medRxiv preprint doi: https://doi.org/10.1101/2021.11.07.21266025; this version posted November 8,2021 . The copyright holder for this preprint (which was not certified by peer review) is the author/funder, who has granted medRxiv a license to display the preprint in

All rights reserved. No reuse allowed without permission.

\title{
Predictions of arrhythmic, heart failure and mortality outcomes in pericarditis using
}

\section{automatic electrocardiogram analysis}

Ishan Lakhani * 1, Jiandong Zhou *2, Michelle Vangi Wong ${ }^{1}$, Sharen Lee ${ }^{1,3}$, Jovy Ka Yiu Lau $\mathrm{BSc}^{1}$, Silverdew Shi ${ }^{1}$, Wing Tak Wong $\mathrm{PhD}^{4}$, Tong Liu MD PhD ${ }^{5}$, Qingpeng Zhang $\mathrm{PhD}$ *2,

$$
\text { Gary Tse PhD FRCP FFPH * } 4
$$

${ }^{1}$ Cardiovascular Analytics Group, Laboratory of Cardiovascular Physiology, Hong Kong, China

${ }^{2}$ School of Data Science, City University of Hong Kong, Hong Kong, Hong Kong, China

${ }^{3}$ Li Ka Shing Institute of Health Sciences, Hong Kong, China

${ }^{4}$ School of Life Sciences, Chinese University of Hong Kong, Hong Kong S.A.R., China

${ }^{5}$ Tianjin Key Laboratory of Ionic-Molecular Function of Cardiovascular Disease, Department of Cardiology, Tianjin Institute of Cardiology, Second Hospital of Tianjin Medical

University, Tianjin 300211, China

${ }^{6}$ Faculty of Health and Medical Sciences, University of Surrey, GU2 7AL, Guildford, United Kingdom

* equal contributions as first authors

Dr. Gary Tse, PhD, FRCP, FFPH

Tianjin Key Laboratory of Ionic-Molecular Function of Cardiovascular disease, Department

of Cardiology, Tianjin Institute of Cardiology, Second Hospital of Tianjin Medical

University, Tianjin 300211, People's Republic of China, China

Faculty of Health and Medical Sciences, University of Surrey, GU2 7AL, Guildford, United

Kingdom

Email: g.tse@surrey.ac.uk / garytse86@,gmail.com 
medRxiv preprint doi: https://doi.org/10.1101/2021.11.07.21266025; this version posted November 8, 2021. The copyright holder for this preprint (which was not certified by peer review) is the author/funder, who has granted medRxiv a license to display the preprint in All rights reserved. No reuse allowed without permission.

Dr. Qingpeng Zhang, PhD

School of Data Science, City University of Hong Kong,

Hong Kong SAR, China

Email: qingpeng.zhang@,cityu.edu.hk 
medRxiv preprint doi: https://doi.org/10.1101/2021.11.07.21266025; this version posted November 8,2021 . The copyright holder for this preprint (which was not certified by peer review) is the author/funder, who has granted medRxiv a license to display the preprint in All rights reserved. No reuse allowed without permission.

\begin{abstract}
Background:

Pericarditis is a relatively rare disease with a global burden. Despite its strong association with adverse cardiovascular outcomes, identification of patients at risk of future heart failure or arrhythmic events is difficult. In the following study, automated electrocardiogram (ECG) were used to predict new onset ventricular tachycardia/fibrillation (VT/VF), atrial fibrillation (AF) and heart failure with reduced ejection fraction (HF) in an Asian cohort of pericarditis patients.
\end{abstract}

Methods: Consecutive patients admitted to a single tertiary center in Hong Kong, China, for a diagnosis of pericarditis between 1st January 2005 and 31st December 2019 with baseline ECG measurements were included. Patients with existing AF or HF were excluded. The follow-up period was until the 31 st December 2020, or death. Cox regression was applied to identify significant predictors of the primary outcomes (incident VT/VF, AF or HF).

Results: A total of 874 patients were included. The cohort was $57 \%$ male and had a median age of 59 (IQR: 50-70) years old. During follow-up, 57 patients (6.5\%), $156(17.8 \%)$ and 168 (19.2\%) suffered from VT/VF, AF and HF, respectively. Cox regression identified baseline $\mathrm{VT} / \mathrm{VF}$, terminal angle of the QRS vector in the transverse plane, mean QRS duration and mean QTc intervals as significant predictors of incident VT/VF events, with only the former most maintaining significance in multivariate analysis. In contrast, baseline age, prior diagnoses of hypertension, initial angle and magnitude of the QRS vector in the transverse 
medRxiv preprint doi: https://doi.org/10.1101/2021.11.07.21266025; this version posted November 8, 2021. The copyright holder for this preprint (which was not certified by peer review) is the author/funder, who has granted medRxiv a license to display the preprint in All rights reserved. No reuse allowed without permission.

plane, P-wave and QRS axis in the frontal plane, ST segment axis in the frontal and horizontal planes, mean PT interval, mean PR segment duration and QTc intervals were all univariate predictors of incident AF, albeit only baseline age and initial angel of the QRS vector in the transverse plane retained significance after multivariate adjustment. As it pertains to new-onset HF, several clinical and electrocardiographic parameters demonstrated an association with HF in univariate analysis, with prior diagnosis of HT or DM, initial QRS angle in transverse plane, I 40 in horizontal axis, ST-segment axis in the horizontal plane, T-wave frontal axis and atrial rate, of which, except for prior diagnosis of DM, I40 in horizontal axis and T-wave frontal axis, all variables showcased significant relationships in multivariate analysis

Interpretation: $\mathrm{AF}$ and $\mathrm{HF}$ are relatively common complications $\mathrm{VT} / \mathrm{VF}$ occurs less frequently in the context of pericarditis. Different clinical and ECG predictors of these outcomes were identified. Future studies are still needed to evaluate their use for risk stratification in the clinical setting. 
medRxiv preprint doi: https://doi.org/10.1101/2021.11.07.21266025; this version posted November 8,2021 . The copyright holder for this preprint (which was not certified by peer review) is the author/funder, who has granted medRxiv a license to display the preprint in

All rights reserved. No reuse allowed without permission.

\section{Introduction}

Acute pericarditis, an inflammation of the pericardial layer of the heart, is an immune-mediated clinical condition that is often unrecognized and associated with numerous of complications 1. Previous cohort studies have explored the aetiology ${ }^{2}$, clinical presentation ${ }^{3,4}$ and risk factors leading to readmission ${ }^{5}$, composite of cardiac tamponade, constrictive pericarditis, failed therapy, recurrence and mortality ${ }^{6}$. By contrast, few studies have explored atrial or ventricular arrhythmic outcomes. Moreover, recent studies have reported the roles of automated electrocardiogram (ECG) analysis to facilitate risk stratification and prediction. However, few studies have examined the roles of ECG predictors for adverse outcomes in the pericarditis population. Therefore, the aim of this study is to investigate the predictive roles of automated electrocardiograms (ECG) for predicting new onset atrial fibrillation (AF), ventricular tachycardia/fibrillation (VT/VF) or heart failure (HF) in a cohort of pericarditis patients admitted to a single tertiary centre.

\section{Methods}

Study design and population

The study was approved by The Joint Chinese University of Hong Kong - New Territories East Cluster Clinical Research Ethics Committee. This was a retrospective, territory-wide cohort study of pericarditispatients with baseline ECG measurementsfrom $1^{\text {st }}$ January 2005 to $31^{\text {st }}$ December2019admitted to a single tertiary center fromthe Hong Kong region of China. The patients were identified from the Clinical Data Analysis and Reporting System (CDARS), a 
medRxiv preprint doi: https://doi.org/10.1101/2021.11.07.21266025; this version posted November 8,2021 . The copyright holder for this preprint (which was not certified by peer review) is the author/funder, who has granted medRxiv a license to display the preprint in

All rights reserved. No reuse allowed without permission.

territory-wide database that centralizes patient information from 43 local hospitals and their associated ambulatory and outpatient facilities to establish comprehensive medical data, including clinical characteristics, disease diagnosis, laboratory results, and drug treatment details. The system has been previously used by both our team and other teams in Hong Kong $^{7,8}$. Patients'demographics, priorcomorbidities, medication prescriptions, laboratory examinations of complete blood counts, biochemical liver and renal tests, lipid and glucose tests and cardiac function tests, and ECG measurements were extracted.The list of ICD-9 codes for comorbidities and outcomes were detailed in the Supplementary Table 1.

\section{Baseline characteristics and ECG measurements}

Clinical data was extracted from electronic health records. The following baseline clinical data were collected: 1) sex; 2) age of initial Brugada pattern presentation; 3) follow-up period; 4) type of Brugada pattern and presence of fever at initial presentation; 5) family history of $\mathrm{BrS}$ and $\mathrm{VF} / \mathrm{SCD}$; 6) manifestation of syncope and if present, the number of episodes; 7) manifestation of VT/VF and if present, the number of episodes; 8) sodium channel blocker challenge test and results; 9) concomitant presence of other arrhythmia; 10) implantation of ICD. Patients presented with two or more episodes of VT/VF were defined to be of high VT/VF burden. Automatically measured parameters from ECG related to the P, Q, $\mathrm{R}, \mathrm{S}$ and T-wave were extracted. This has been done previously by our team and further details could be found here ${ }^{9,10}$. 
medRxiv preprint doi: https://doi.org/10.1101/2021.11.07.21266025; this version posted November 8,2021 . The copyright holder for this preprint (which was not certified by peer review) is the author/funder, who has granted medRxiv a license to display the preprint in

All rights reserved. No reuse allowed without permission.

\section{Outcomes and statistical analysis}

The primary outcome was new onset VT/VF. Secondary outcomes included new onset 1) AF, 2) new-onset HFrEF and 3) all-cause mortality. The secondary outcomes were cardiovascular mortality and all-cause mortality. Mortality data were obtained from the Hong Kong Death Registry, a population-based official government registry with the registered death records of all Hong Kong citizens linked to CDARS. There was no adjudication of the outcomes as this relied on the ICD-9 coding or a record in the death registry. However, the coding was performed by the clinicians or administrative staff, who were not involved in the mode development. Descriptive statistics are used to summarize baseline clinical characteristics of all pericarditis patients and based on the occurrence of the primary and secondary outcomes. Continuous variables were presented as median (95\% confidence interval[CI] or interquartile range[IQR]) and categorical variables were presented as count (\%). The Mann-Whitney $U$ test was used to compare continuous variables. The $\chi 2$ test with Yates' correction was used for $2 \times 2$ contingency data. Univariate logistic regression identifies significant mortality risk predictors. Hazard ratios (HRs) with corresponding 95\% CIs and P-values were reported. There was no imputation performed for missing data. No blinding was performed for the predictor as the values were obtained from the electronic health records automatically.All statistical tests were two-tailed and considered significant if $p$ value $<0.001$. They were performed using RStudio software (Version: 1.1.456) and Python (Version: 3.6). 
medRxiv preprint doi: https://doi.org/10.1101/2021.11.07.21266025; this version posted November 8,2021 . The copyright holder for this preprint (which was not certified by peer review) is the author/funder, who has granted medRxiv a license to display the preprint in All rights reserved. No reuse allowed without permission.

\section{Results}

Basic characteristics

The baseline clinical characteristics of patients with/without spontaneous VT/VF are presented in Table 1. Subjects who developed incident VT/VF tended to have baseline VT/VF prior to the diagnosis of pericarditis. Regarding blood parameters, these individuals also reported significantly higher eosinophil counts, low-density-lipoprotein (LDL) levels, high-sensitivity troponin I (hsTnI) levels and lactate dehydrogenase (LDH) levels, as well as a lower mean corpuscular volume (MCV). Patients who developed VT/VF likewise had greater terminal QRS angles in the transverse plane and T-wave frontal axis, along with a longer QTc interval.

\section{Predictors of primary and secondary outcomes}

A total of 874 patients were included. The cohort was $57 \%$ male and had a median age of 59 (IQR: 50-70) years old. During follow-up, 57 patients (6.5\%), $156(17.8 \%)$ and $168(19.2 \%)$ suffered from VT/VF, AF and HF, respectively. Cox regression identified baseline VT/VF, terminal angle of the QRS vector in the transverse plane, mean QRS duration and mean QTc intervals as significant predictors of incident VT/VF events, with only the former most maintaining significance in multivariate analysis (HR: 5.03; 95\% CI: $1.12-22.6 ; \mathrm{P}=0.035$ ). In contrast, regarding secondary outcomes, baseline age, prior diagnoses of hypertension, initial angle and magnitude of the QRS vector in the transverse plane, P-wave and QRS axis in the frontal plane, ST segment axis in the frontal and horizontal planes, mean PT interval, 
medRxiv preprint doi: https://doi.org/10.1101/2021.11.07.21266025; this version posted November 8,2021 . The copyright holder for this preprint (which was not certified by peer review) is the author/funder, who has granted medRxiv a license to display the preprint in

All rights reserved. No reuse allowed without permission.

mean PR segment duration and QTc intervals were all univariate predictors of incident AF, albeit only baseline age (HR: 1.03; 95\% CI: $1.02-1.05 ; \mathrm{P}<0.001)$ and initial angle of the QRS vector in the transverse plane (HR: 1.003; 95\% CI: $1.001-1.005 ; \mathrm{P}=0.003)$ retained significance after multivariate adjustment. As it pertains to new-onset HF, several clinical and electrocardiographic parameters demonstrated an association with $\mathrm{HF}$ in univariate analysis, including prior diagnosis of HT (HR: 3.27; 95\% CI: $2.08-5.13$; P $<0.001$ ) or DM, initial QRS angle in transverse plane (HR: 1.002; 95\% CI: $1.001-1.004 ; \mathrm{P}=0.008)$, I 40 in horizontal axis, ST-segment axis in the horizontal plane (HR: 1.004; 95\% CI: $1.001-1.009$; $\mathrm{P}=0.008)$, T-wave frontal axis and atrial rate (HR: 1.01; 95\% CI: $1.006-1.014 ; \mathrm{P}<0.001)$, of which, except for prior diagnosis of DM, I40 in horizontal axis and T-wave frontal axis, all variables showcased significant relationships in multivariate analysis.

A total of 396 (45\%) patients died during follow-up, of which 52 (6\%) were cardiovascular-related. Univariate predictors for all-cause mortality comprised baseline age, prior diagnosis of hypertension, terminal and maximum magnitude of the QRS vector in the transverse plane, $\mathrm{T}$ wave $(40 \mathrm{~ms})$ horizontal axis, ventricular rate mean QTc interval, mean PT interval, mean PR segment and QT interval dispersion. Subsequent multivariate adjustment revealed only significant associations for only baseline age (HR: $1.02 ; 95 \% \mathrm{CI}$ : 1.01-1.03; $\mathrm{P}<0.001)$ and maximum magnitude of the QRS vector in the transverse plane (HR: 0.55; 95\% CI: 0.4-0.75; P <0.001). 
medRxiv preprint doi: https://doi.org/10.1101/2021.11.07.21266025; this version posted November $8,2021$. The copyright holder for this preprint (which was not certified by peer review) is the author/funder, who has granted medRxiv a license to display the preprint in

All rights reserved. No reuse allowed without permission.

\section{Discussion}

The major limitation of this study is that it is based on a single centre cohort. We recognize that the derivation populations may differ from other populations based both on hospital conditions and inherent demographic differences. An external validation using independent external cohort allows us to assess the broader clinical utility of our findings. However, the model should be further externally validated using patient data from other regions.

\section{Conflicts of Interest}

None.

\section{References}

1 Chiabrando, J. G. et al. Management of Acute and Recurrent Pericarditis: JACC State-of-the-Art Review. J Am Col/ Cardio/75, 76-92, doi:10.1016/j.jacc.2019.11.021 (2020).

2 Sathirareuangchai, S., Kobayashi, M. \& Shimizu, D. Etiologies of pericarditis in hospital and forensic autopsies. Cardiovasc Patho/49, 107262, doi:10.1016/j.carpath.2020.107262 (2020).

3 Imazio, M. et al. Myopericarditis versus viral or idiopathic acute pericarditis. Heart94, 498-501, doi:10.1136/hrt.2006.104067 (2008).

4 Kyto, V., Sipila, J. \& Rautava, P. Clinical profile and influences on outcomes in patients hospitalized for acute pericarditis. Circulation130, 1601-1606, doi:10.1161/CIRCULATIONAHA.114.010376 (2014).

5 Sreenivasan, J. et al. Rate, Causes, and Predictors of 30-Day Readmission Following Hospitalization for Acute Pericarditis. Am J Med133, 1453-1459 e1451, doi:10.1016/j.amjmed.2020.05.027 (2020).

6 Vecchie, A. et al. Clinical Presentation and Outcomes of Acute Pericarditis in a Large Urban Hospital in the United States of America. Chest158, 2556-2567, doi:10.1016/j.chest.2020.07.039 (2020).

7 Zhou, J. et al. Proton pump inhibitor or famotidine use and severe COVID-19 disease: a propensity score-matched territory-wide study. Gut, doi:10.1136/gutjnl-2020-323668 (2020). 
medRxiv preprint doi: https://doi.org/10.1101/2021.11.07.21266025; this version posted November 8, 2021. The copyright holder for this preprint (which was not certified by peer review) is the author/funder, who has granted medRxiv a license to display the preprint in All rights reserved. No reuse allowed without permission.

8 Lee, S. et al. Glycemic and lipid variability for predicting complications and mortality in diabetes mellitus using machine learning. BMC Endocr Disord21, 94, doi:10.1186/s12902-021-00751-4 (2021).

9 Tse, G. et al. P-Wave Area Predicts New Onset Atrial Fibrillation in Mitral Stenosis: A Machine Learning Approach. Front Bioeng Biotechno/8, 479, doi:10.3389/fbioe.2020.00479 (2020).

10 Tse, G. et al. Automated Electrocardiogram Analysis Identifies Novel Predictors of Ventricular Arrhythmias in Brugada Syndrome. Frontiers in Cardiovascular Medicine7, 399 (2021). 
Table 1. Baseline clinical characteristics of patients with/without spontaneous VT/VF.

$*$ for $\mathrm{p} \leq 0.05, * *$ for $\mathrm{p} \leq 0.01, * * *$ for $\mathrm{p} \leq 0.001$.

\# indicates that the comparisons were made between patients with/without spontaneous VT/VF.

\begin{tabular}{|c|c|c|c|c|}
\hline Characteristics & $\begin{array}{l}\text { All patients }(\mathrm{N}=874) \\
\text { Median }(\mathrm{IQR}) ; \mathrm{N} \text { or } \operatorname{Count}(\%)\end{array}$ & $\begin{array}{l}\text { Spontaneous VT/VF }(\mathrm{N}=57) \\
\text { Median }(\mathrm{IQR}) ; \mathrm{N} \text { or Count }(\%)\end{array}$ & $\begin{array}{l}\text { No spontaneous VT/VF }(\mathrm{N}=817) \\
\text { Median }(\mathrm{IQR}) ; \mathrm{N} \text { or } \operatorname{Count}(\%)\end{array}$ & P value ${ }^{\#}$ \\
\hline \multicolumn{5}{|l|}{ Demographics } \\
\hline Male gender & $494(56.52 \%)$ & $30(52.63 \%)$ & $464(56.79 \%)$ & 0.8325 \\
\hline Female gender & $380(43.47 \%)$ & $27(47.36 \%)$ & $353(43.20 \%)$ & 0.7972 \\
\hline Baseline age, years & $59.43(49.5-69.76) ; \mathrm{n}=874$ & $58.84(47.25-68.06) ; \mathrm{n}=57$ & $59.51(49.82-69.86) ; n=817$ & 0.477 \\
\hline$<40$ & $119(13.61 \%)$ & $12(21.05 \%)$ & $107(13.09 \%)$ & 0.2144 \\
\hline$[40,50)$ & $105(12.01 \%)$ & $6(10.52 \%)$ & $99(12.11 \%)$ & 0.9139 \\
\hline$[50-60)$ & $221(25.28 \%)$ & $13(22.80 \%)$ & $208(25.45 \%)$ & 0.8468 \\
\hline$[60-70)$ & $216(24.71 \%)$ & $14(24.56 \%)$ & $202(24.72 \%)$ & 0.8946 \\
\hline$[70-80)$ & $145(16.59 \%)$ & $10(17.54 \%)$ & $135(16.52 \%)$ & 0.9902 \\
\hline$>=80$ & $68(7.78 \%)$ & $2(3.50 \%)$ & $66(8.07 \%)$ & 0.3607 \\
\hline $\begin{array}{l}\text { Charlson standard } \\
\text { comorbidity index }\end{array}$ & $2.0(1.0-4.0) ; \mathrm{n}=874$ & $2.0(0.0-4.0) ; \mathrm{n}=57$ & $2.0(1.0-4.0) ; \mathrm{n}=817$ & 0.3003 \\
\hline \multicolumn{5}{|l|}{ Past comorbidities } \\
\hline Baseline VT/VF & $8(0.91 \%)$ & $4(7.01 \%)$ & $4(0.48 \%)$ & $<0.0001 * * *$ \\
\hline Cerebrovascular disease & $28(3.20 \%)$ & $2(3.50 \%)$ & $26(3.18 \%)$ & 0.7969 \\
\hline Rheumatic disease & $22(2.51 \%)$ & $4(7.01 \%)$ & $18(2.20 \%)$ & 0.0862 \\
\hline Renal diseases & $48(5.49 \%)$ & $3(5.26 \%)$ & $45(5.50 \%)$ & 0.821 \\
\hline Diabetes mellitus & $25(2.86 \%)$ & $3(5.26 \%)$ & $22(2.69 \%)$ & 0.4992 \\
\hline Hypertension & $155(17.73 \%)$ & $11(19.29 \%)$ & $144(17.62 \%)$ & 0.9281 \\
\hline AMI & $23(2.63 \%)$ & $0(0.00 \%)$ & $23(2.81 \%)$ & 0.405 \\
\hline
\end{tabular}




\begin{tabular}{|c|c|c|c|c|}
\hline COPD & $8(0.91 \%)$ & $1(1.75 \%)$ & $7(0.85 \%)$ & 0.9702 \\
\hline IHD & $86(9.83 \%)$ & $6(10.52 \%)$ & $80(9.79 \%)$ & 0.9477 \\
\hline PVD & $12(1.37 \%)$ & $1(1.75 \%)$ & $11(1.34 \%)$ & 0.7372 \\
\hline Stroke/TIA & $25(2.86 \%)$ & $2(3.50 \%)$ & $23(2.81 \%)$ & 0.9082 \\
\hline Gastrointestinal bleeding & $39(4.46 \%)$ & $2(3.50 \%)$ & $37(4.52 \%)$ & 0.9892 \\
\hline $\begin{array}{l}\text { Immune mediated } \\
\text { enterocolitis }\end{array}$ & $47(5.37 \%)$ & $2(3.50 \%)$ & $45(5.50 \%)$ & 0.7547 \\
\hline $\begin{array}{l}\text { Anxiety disorder and } \\
\text { depression }\end{array}$ & $17(1.94 \%)$ & $1(1.75 \%)$ & $16(1.95 \%)$ & 0.6962 \\
\hline SCD & $25(2.86 \%)$ & $1(1.75 \%)$ & $24(2.93 \%)$ & 0.9257 \\
\hline Atrial flutter & $6(0.68 \%)$ & $0(0.00 \%)$ & $6(0.73 \%)$ & 0.853 \\
\hline Accident fall & $50(5.72 \%)$ & $3(5.26 \%)$ & $47(5.75 \%)$ & 0.8812 \\
\hline Malignancy & $165(18.87 \%)$ & $8(14.03 \%)$ & $157(19.21 \%)$ & 0.523 \\
\hline Metastatic solid tumor & $94(10.75 \%)$ & $2(3.50 \%)$ & $92(11.26 \%)$ & 0.1429 \\
\hline \multicolumn{5}{|l|}{ Medications } \\
\hline ACEI & $77(8.81 \%)$ & $7(12.28 \%)$ & $70(8.56 \%)$ & 0.5317 \\
\hline $\mathrm{ARB}$ & $30(3.43 \%)$ & $5(8.77 \%)$ & $25(3.05 \%)$ & 0.0728 \\
\hline Lipid-lowering drugs & - & - & - & - \\
\hline Anticoagulants & $100(11.44 \%)$ & $11(19.29 \%)$ & $89(10.89 \%)$ & 0.145 \\
\hline Insulin & - & - & - & - \\
\hline Incretin mimetics & $10(1.14 \%)$ & $1(1.75 \%)$ & $9(1.10 \%)$ & 0.8411 \\
\hline \multicolumn{5}{|l|}{ Complete blood counts } \\
\hline $\begin{array}{l}\text { Mean corpuscular volume, } \\
\text { fL }\end{array}$ & $89.8(86.3-93.35) ; \mathrm{n}=543$ & $88.5(85.0-90.6) ; \mathrm{n}=37$ & $90.0(86.4-93.5) ; \mathrm{n}=506$ & $0.0367 *$ \\
\hline Eosinophil, x10^9/L & $0.1(0.0-0.2) ; \mathrm{n}=504$ & $0.2(0.1-0.3) ; \mathrm{n}=33$ & $0.1(0.0-0.2) ; \mathrm{n}=471$ & $0.0008 * * *$ \\
\hline
\end{tabular}


Lymphocyte, $\mathrm{x} 10^{\wedge} 9 / \mathrm{L}$

$1.15(0.8-1.7) ; \mathrm{n}=505$

Monocyte, $\mathrm{x} 10^{\wedge} 9 / \mathrm{L}$

$0.6(0.4-0.8) ; \mathrm{n}=505$

Neutrophil, x10^9/L

$4.9(3.4-7.7) ; \mathrm{n}=505$

White cell count, $x 10^{\wedge} 9 / \mathrm{L} \quad 7.0(5.5-9.15) ; \mathrm{n}=543$

Mean cell haemoglobin, pg 30.6(29.0-32.0);n=543

Platelet, $\mathrm{x} 10^{\wedge} 9 / \mathrm{L}$

$212.0(166.5-275.0) ; \mathrm{n}=543$

Red blood count, $x 10^{\wedge} 12 / \mathrm{L} \quad 4.09(3.6-4.58) ; \mathrm{n}=543$

\section{Liver and renal functions}

$\mathrm{K} /$ Potassium, mmol/L

$4.0(3.7-4.4) ; \mathrm{n}=684$

Urate, $\mathrm{mmol} / \mathrm{L}$

Albumin, $\mathrm{g} / \mathrm{L}$

$0.38(0.28-0.49) ; \mathrm{n}=100$

$35.9(30.5-40.0) ; \mathrm{n}=681$

$\mathrm{Na} /$ Sodium, mmol/L

$138.45(135.2-140.9) ; \mathrm{n}=684$

Urea, $\mathrm{mmol} / \mathrm{L}$

$5.8(4.26-8.41) ; \mathrm{n}=683$

Protein, $\mathrm{g} / \mathrm{L}$

$71.0(65.6-75.4) ; \mathrm{n}=681$

Creatinine, umol/L

\begin{abstract}
$84.0(66.0-113.0) ; \mathrm{n}=682$
\end{abstract}
Alkaline phosphatase, U/L $\quad 82.0(65.0-118.0) ; \mathrm{n}=681$

Aspartate transaminase, U/L 29.5(18.0-58.1); $\mathrm{n}=82$

Alanine transaminase, $\mathrm{U} / \mathrm{L} \quad 24.0(16.0-44.0) ; \mathrm{n}=681$

Bilirubin, umol/L

$10.4(6.9-16.8) ; \mathrm{n}=681$

\section{Lipid and glucose tests}

Triglyceride, mmol/L

Low-density lipoprotein, $\mathrm{mmol} / \mathrm{L}$

1.01(0.75-1.44);n=167

$2.22(1.61-2.86) ; \mathrm{n}=153$

High-density lipoprotein, $\mathrm{mmol} / \mathrm{L}$

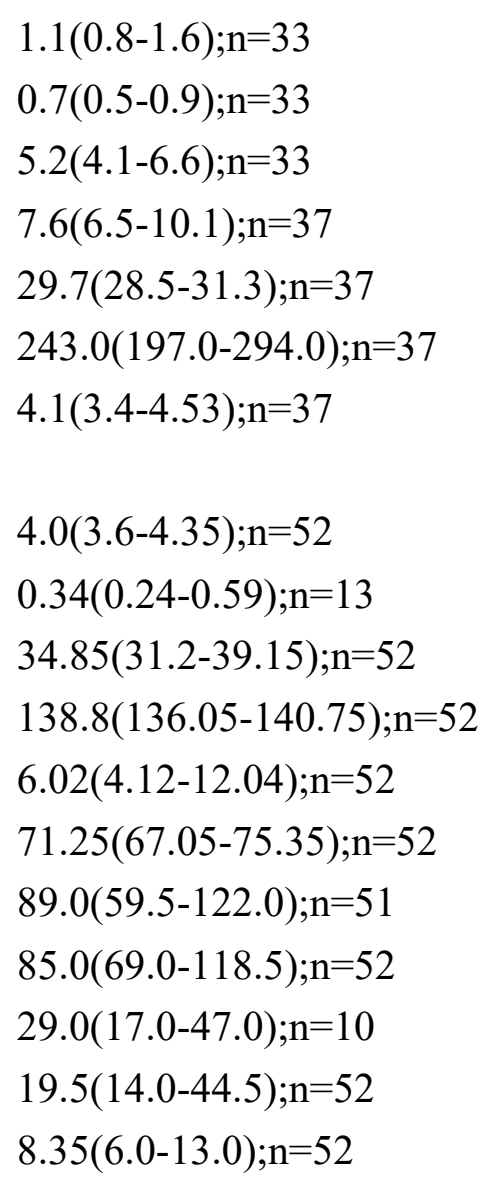

$1.15(0.8-1.7) ; \mathrm{n}=472$

$$
0.6(0.4-0.77) ; \mathrm{n}=472
$$

$4.9(3.4-7.75) ; \mathrm{n}=472$

6.95(5.5-9.0); $\mathrm{n}=506$

$30.7(29.1-32.1) ; \mathrm{n}=506$

$209.0(165.0-274.0) ; \mathrm{n}=506$

$4.08(3.6-4.58) ; \mathrm{n}=506$

4.0(3.7-4.4); $\mathrm{n}=632$

$0.38(0.28-0.47) ; \mathrm{n}=87$

$35.9(30.5-40.1) ; \mathrm{n}=629$

$138.4(135.05-140.9) ; \mathrm{n}=632$

$5.73(4.27-8.4) ; \mathrm{n}=631$

$71.0(65.5-75.4) ; \mathrm{n}=629$

$83.0(66.0-111.5) ; \mathrm{n}=631$

$82.0(64.0-118.0) ; \mathrm{n}=629$

$29.5(18.0-62.5) ; \mathrm{n}=72$

$24.0(16.0-44.0) ; \mathrm{n}=629$

$10.6(7.0-17.0) ; \mathrm{n}=629$

$1.0(0.74-1.42) ; \mathrm{n}=154$

$2.22(1.62-2.84) ; \mathrm{n}=142$

$1.12(0.82-1.48) ; \mathrm{n}=142$
0.9463

0.0509

0.5897

0.056

0.0608

0.0958

0.6528

0.5585

0.7701

0.6382

0.7475

0.6471

0.9386

0.7487

0.4949

0.6967

0.1168

0.0766

0.0864

0.9859

0.0273* 
Cholesterol, $\mathrm{mmol} / \mathrm{L}$

HbA1c, g/dL

Glucose, $\mathrm{mmol} / \mathrm{L}$

Cardiac, clotting,

inflammatory,

and acid-base tests

D-dimer, $\mathrm{ng} / \mathrm{mL}$

High sensitive troponin-I,

$\mathrm{ng} / \mathrm{L}$

Lactate dehydrogenase, U/L 265.0(211.0-387.5);n=483

APTT, second

Prothrombin time/INR,

second

C-reactive protein, $\mathrm{mg} / \mathrm{dL} \quad 2.91(0.56-8.62) ; \mathrm{n}=556$

\section{ECG measurements}

Trans QRS initangle

Trans QRS initmag

Trans QRS max angle

Trans QRS max mag

Trans QRS term angle

Trans QRS term mag

Trans QRS cwrot

$\mathrm{P}$ wave front axis

$P$ wave horizontal axis

I 40 front axis
$33.0(30.2-36.6) ; \mathrm{n}=721$

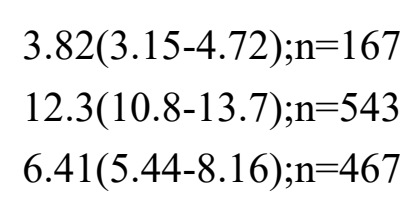

$0.02(0.01-0.07) ; \mathrm{n}=444$

$11.9(10.8-13.7) ; \mathrm{n}=721$

$42.0(19.0-90.0) ; \mathrm{n}=574$

$0.31(0.18-0.5) ; \mathrm{n}=574$

$331.0(42.5-347.0) ; \mathrm{n}=574$

$1.23(0.86-1.66) ; \mathrm{n}=574$

243.0(214.0-273.0); $\mathrm{n}=574$

$0.3(0.2-0.41) ; \mathrm{n}=574$

$-100.0(-100.0--87.5) ; \mathrm{n}=574$

$54.0(39.0-68.0) ; \mathrm{n}=798$

20.0(4.0-37.0); $\mathrm{n}=830$
I 40 front axis

\begin{abstract}
$38.0(15.0-58.0) ; \mathrm{n}=860$
\end{abstract}

$$
\begin{aligned}
& 4.05(3.42-4.82) ; \mathrm{n}=13 \\
& 11.4(10.6-13.7) ; \mathrm{n}=37 \\
& 6.65(5.73-7.9) ; \mathrm{n}=36
\end{aligned}
$$
$3.82(3.15-4.71) ; \mathrm{n}=154$
$12.3(10.8-13.7) ; \mathrm{n}=506$
6.4(5.43-8.2);n=431

0.9167

0.3974

0.5478

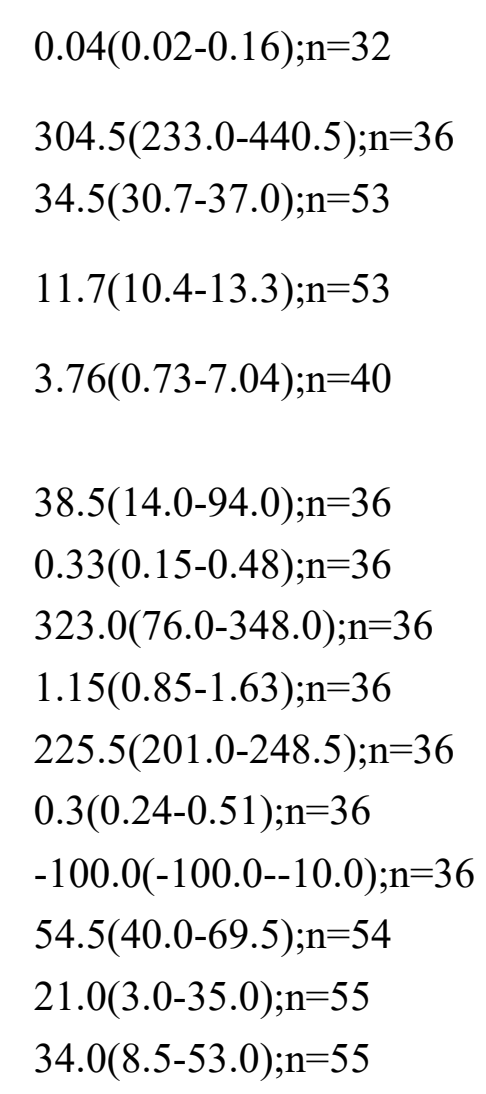

2684.52(1164.69-6470.84);n=64

0.9131

$$
\text { 0.02(0.01-0.07); } \mathrm{n}=412
$$

$0.0155^{*}$

$262.0(207.0-371.5) ; \mathrm{n}=447$

$33.0(30.2-36.45) ; \mathrm{n}=668$

0.0428*

0.1871

11.9(10.8-13.7); $\mathrm{n}=668$

0.4269

$2.78(0.56-8.67) ; \mathrm{n}=516$

0.6553

42.0(19.0-90.0); $\mathrm{n}=538$

0.5997

$0.31(0.18-0.5) ; \mathrm{n}=538$

0.8278

0.5825

0.4962

$0.0183 *$

0.2255

0.3281

0.6899

0.7535

0.177 


\begin{tabular}{|c|c|c|c|c|}
\hline I 40 horizon axis & $28.0(11.5-44.0) ; \mathrm{n}=839$ & $28.0(17.0-48.5) ; \mathrm{n}=55$ & $28.0(11.0-44.0) ; \mathrm{n}=784$ & 0.4206 \\
\hline QRS front axis & $52.0(20.0-75.0) ; \mathrm{n}=871$ & $48.0(10.0-79.0) ; \mathrm{n}=57$ & $52.0(21.0-75.0) ; \mathrm{n}=814$ & 0.7057 \\
\hline QRS horizon axis & $-11.0(-30.0-9.0) ; \mathrm{n}=853$ & $-8.0(-46.5-20.0) ; \mathrm{n}=56$ & $-11.0(-30.0-8.0) ; \mathrm{n}=797$ & 0.8747 \\
\hline $\mathrm{T}$ wave $40 \mathrm{~ms}$ front axis & $69.0(19.0-101.5) ; \mathrm{n}=862$ & $84.0(8.0-118.0) ; \mathrm{n}=57$ & $69.0(19.0-100.0) ; \mathrm{n}=805$ & 0.2919 \\
\hline $\mathrm{T}$ wave $40 \mathrm{~ms}$ horizon axis & $-23.0(-52.0-184.0) ; \mathrm{n}=829$ & $-10.5(-55.0-211.0) ; \mathrm{n}=56$ & $-23.0(-52.0-179.0) ; \mathrm{n}=773$ & 0.5137 \\
\hline ST segment front axis & $63.0(30.0-113.5) ; \mathrm{n}=827$ & $72.0(33.5-126.0) ; \mathrm{n}=56$ & $63.0(30.0-112.5) ; \mathrm{n}=771$ & 0.5575 \\
\hline ST segment horizon axis & $76.0(50.0-118.0) ; \mathrm{n}=780$ & $79.0(51.0-143.5) ; \mathrm{n}=51$ & $76.0(50.0-117.0) ; \mathrm{n}=729$ & 0.4827 \\
\hline $\mathrm{T}$ wave front axis & $45.0(14.0-72.0) ; \mathrm{n}=857$ & $50.0(35.0-101.0) ; \mathrm{n}=55$ & $44.0(13.0-70.5) ; \mathrm{n}=802$ & $0.0322 *$ \\
\hline T wave horizon axis & $46.0(25.0-76.0) ; \mathrm{n}=805$ & $48.0(16.0-84.0) ; \mathrm{n}=53$ & $46.0(25.0-75.5) ; \mathrm{n}=752$ & 0.8192 \\
\hline Atrial rate & $80.0(67.0-99.5) ; \mathrm{n}=874$ & $83.0(75.0-92.0) ; \mathrm{n}=57$ & $80.0(66.0-100.0) ; \mathrm{n}=817$ & 0.6285 \\
\hline Mean ventricular rate & $81.0(68.0-97.0) ; \mathrm{n}=874$ & $83.0(76.0-91.0) ; \mathrm{n}=57$ & $80.0(68.0-97.0) ; \mathrm{n}=817$ & 0.3177 \\
\hline Mean PT interval & $161.0(144.5-177.5) ; \mathrm{n}=874$ & $160.0(148.0-178.0) ; \mathrm{n}=57$ & $161.0(144.0-177.0) ; \mathrm{n}=817$ & 0.649 \\
\hline Mean PR segment & $63.0(48.0-78.0) ; \mathrm{n}=874$ & $64.0(48.0-78.0) ; \mathrm{n}=57$ & $63.0(49.0-78.0) ; \mathrm{n}=817$ & 0.8753 \\
\hline Mean QRS duration & $85.0(78.0-95.0) ; \mathrm{n}=874$ & $86.0(81.0-100.0) ; \mathrm{n}=57$ & $85.0(78.0-94.0) ; \mathrm{n}=817$ & 0.0889 \\
\hline Mean QT interval & $377.0(345.0-408.5) ; \mathrm{n}=874$ & $380.0(348.0-415.0) ; \mathrm{n}=57$ & $377.0(344.0-408.0) ; \mathrm{n}=817$ & 0.5507 \\
\hline Mean QTc interval & $433.0(413.0-458.0) ; \mathrm{n}=874$ & $443.0(424.0-464.0) ; \mathrm{n}=57$ & $433.0(412.0-458.0) ; \mathrm{n}=817$ & $0.0202 *$ \\
\hline QT interval dispersion & $64.0(0.0-104.0) ; \mathrm{n}=874$ & $60.0(0.0-104.0) ; \mathrm{n}=57$ & $64.0(0.0-104.0) ; n=817$ & 0.686 \\
\hline
\end{tabular}
activated partial thromboplastin time. 
Table 2. Baseline and clinical characteristics of patients with new onset $\mathrm{HF}$ and new onset $\mathrm{AF}$.

* for $\mathrm{p} \leq 0.05, * *$ for $\mathrm{p} \leq 0.01, * * *$ for $\mathrm{p} \leq 0.001$.

\begin{tabular}{|c|c|c|c|c|c|c|}
\hline \multirow{2}{*}{$\begin{array}{l}\text { Characterist } \\
\text { ics }\end{array}$} & \multirow{2}{*}{$\begin{array}{l}\text { New onset HF }(\mathrm{N}=168) \\
\text { Median (IQR);N or } \\
\text { Count(\%) }\end{array}$} & \multicolumn{2}{|l|}{ No new onset HF $(N=706)$} & \multirow{2}{*}{$\begin{array}{l}\text { New onset AF }(\mathrm{N}=156) \\
\text { Median (IQR); N or } \\
\text { Count }(\%)\end{array}$} & \multicolumn{2}{|l|}{ No new onset $A F(N=718)$} \\
\hline & & $\begin{array}{l}\text { Median (IQR);N or } \\
\text { Count(\%) }\end{array}$ & P value & & $\begin{array}{l}\text { Median (IQR); N or } \\
\text { Count }(\%)\end{array}$ & P value \\
\hline \multirow{2}{*}{\multicolumn{7}{|c|}{$\begin{array}{l}\text { Demographi } \\
\text { cs }\end{array}$}} \\
\hline & & & & & & \\
\hline Male gender & $92(54.76 \%)$ & $402(56.94 \%)$ & 0.8421 & $86(55.12 \%)$ & $408(56.82 \%)$ & 0.8957 \\
\hline $\begin{array}{l}\text { Female } \\
\text { gender }\end{array}$ & $76(45.23 \%)$ & $304(43.05 \%)$ & 0.8086 & $70(44.87 \%)$ & $310(43.17 \%)$ & 0.8711 \\
\hline $\begin{array}{l}\text { Baseline age, } \\
\text { years }\end{array}$ & $66.33(57.07-77.3) ; \mathrm{n}=168$ & $57.69(47.94-68.03) ; \mathrm{n}=706$ & $\begin{array}{l}<0.0001 * \\
* *\end{array}$ & \multicolumn{2}{|c|}{$66.06(58.12-73.43) ; \mathrm{n}=15657.56(47.22-68.44) ; \mathrm{n}=718$} & $\begin{array}{l}<0.0001 * \\
* *\end{array}$ \\
\hline$<40$ & $10(5.95 \%)$ & $109(15.43 \%)$ & $0.0058 * *$ & $2(1.28 \%)$ & $117(16.29 \%)$ & $\begin{array}{l}<0.0001 * \\
* *\end{array}$ \\
\hline$[40,50)$ & $11(6.54 \%)$ & $94(13.31 \%)$ & $0.0397 *$ & $7(4.48 \%)$ & $98(13.64 \%)$ & $0.0056^{* *}$ \\
\hline$[50-60)$ & $30(17.85 \%)$ & $191(27.05 \%)$ & 0.0642 & $40(25.64 \%)$ & $181(25.20 \%)$ & 0.9909 \\
\hline$[60-70)$ & $49(29.16 \%)$ & $167(23.65 \%)$ & 0.2954 & $54(34.61 \%)$ & $162(22.56 \%)$ & $0.0220^{*}$ \\
\hline$[70-80)$ & $39(23.21 \%)$ & $106(15.01 \%)$ & $0.0438^{*}$ & $35(22.43 \%)$ & $110(15.32 \%)$ & 0.0925 \\
\hline$>=80$ & $29(17.26 \%)$ & $39(5.52 \%)$ & $\begin{array}{l}<0.0001 * \\
* *\end{array}$ & $18(11.53 \%)$ & $50(6.96 \%)$ & 0.109 \\
\hline $\begin{array}{l}\text { Charlson } \\
\text { standard } \\
\text { comorbidity } \\
\text { index }\end{array}$ & $3.0(2.0-4.0) ; \mathrm{n}=168$ & $2.0(1.0-4.0) ; \mathrm{n}=706$ & $\begin{array}{l}0.0005 * * \\
*\end{array}$ & $2.0(2.0-4.0) ; \mathrm{n}=156$ & $2.0(1.0-4.0) ; \mathrm{n}=718$ & $0.0205^{*}$ \\
\hline $\begin{array}{l}\text { Past } \\
\text { comorbiditie }\end{array}$ & & & & & & \\
\hline
\end{tabular}




\begin{tabular}{|c|c|c|}
\hline $\begin{array}{l}\text { Baseline } \\
\text { VT/VF }\end{array}$ & $3(1.78 \%)$ & $5(0.70 \%)$ \\
\hline $\begin{array}{l}\text { Cerebrovascu } \\
\text { lar disease }\end{array}$ & $9(5.35 \%)$ & $19(2.69 \%)$ \\
\hline $\begin{array}{l}\text { Rheumatic } \\
\text { disease }\end{array}$ & $4(2.38 \%)$ & $18(2.54 \%)$ \\
\hline $\begin{array}{l}\text { Renal } \\
\text { diseases }\end{array}$ & $14(8.33 \%)$ & $34(4.81 \%)$ \\
\hline $\begin{array}{l}\text { Diabetes } \\
\text { mellitus }\end{array}$ & $10(5.95 \%)$ & $15(2.12 \%)$ \\
\hline Hypertension & $54(32.14 \%)$ & $101(14.30 \%)$ \\
\hline AMI & $9(5.35 \%)$ & $14(1.98 \%)$ \\
\hline COPD & $3(1.78 \%)$ & $5(0.70 \%)$ \\
\hline IHD & $31(18.45 \%)$ & $55(7.79 \%)$ \\
\hline PVD & $4(2.38 \%)$ & $8(1.13 \%)$ \\
\hline Stroke/TIA & $7(4.16 \%)$ & $18(2.54 \%)$ \\
\hline $\begin{array}{l}\text { Gastrointesti } \\
\text { nal bleeding }\end{array}$ & $10(5.95 \%)$ & $29(4.10 \%)$ \\
\hline $\begin{array}{l}\text { Immune } \\
\text { mediated } \\
\text { enterocolitis }\end{array}$ & $7(4.16 \%)$ & $40(5.66 \%)$ \\
\hline $\begin{array}{l}\text { Anxiety } \\
\text { disorder and }\end{array}$ & $7(4.16 \%)$ & $10(1.41 \%)$ \\
\hline
\end{tabular}

\begin{tabular}{|c|c|c|c|}
\hline 0.3939 & $1(0.64 \%)$ & $7(0.97 \%)$ & 0.9438 \\
\hline 0.1462 & $7(4.48 \%)$ & $21(2.92 \%)$ & 0.4724 \\
\hline 0.8794 & $3(1.92 \%)$ & $19(2.64 \%)$ & 0.8194 \\
\hline 0.1338 & $11(7.05 \%)$ & $37(5.15 \%)$ & 0.487 \\
\hline $0.0206^{*}$ & $6(3.84 \%)$ & $19(2.64 \%)$ & 0.5995 \\
\hline $\begin{array}{l}<0.0001^{*} \\
* *\end{array}$ & $37(23.71 \%)$ & $118(16.43 \%)$ & 0.0972 \\
\hline $0.0355^{*}$ & $4(2.56 \%)$ & $19(2.64 \%)$ & 0.8264 \\
\hline 0.3939 & $1(0.64 \%)$ & $7(0.97 \%)$ & 0.9438 \\
\hline $\begin{array}{l}0.0004 * * \\
*\end{array}$ & $21(13.46 \%)$ & $65(9.05 \%)$ & 0.176 \\
\hline 0.3897 & $3(1.92 \%)$ & $9(1.25 \%)$ & 0.793 \\
\hline 0.4027 & $7(4.48 \%)$ & $18(2.50 \%)$ & 0.3004 \\
\hline 0.4334 & $11(7.05 \%)$ & $28(3.89 \%)$ & 0.1541 \\
\hline 0.5851 & $7(4.48 \%)$ & $40(5.57 \%)$ & 0.7482 \\
\hline 0.0514 & $2(1.28 \%)$ & $15(2.08 \%)$ & 0.7415 \\
\hline
\end{tabular}




\begin{tabular}{|c|c|c|c|c|c|c|}
\hline \multicolumn{7}{|l|}{ depression } \\
\hline $\mathrm{SCD}$ & $9(5.35 \%)$ & $16(2.26 \%)$ & 0.068 & $4(2.56 \%)$ & $21(2.92 \%)$ & 0.9786 \\
\hline Atrial flutter & $2(1.19 \%)$ & $4(0.56 \%)$ & 0.7235 & $5(3.20 \%)$ & $1(0.13 \%)$ & $\begin{array}{l}0.0003 * * \\
*\end{array}$ \\
\hline Accident fall & $11(6.54 \%)$ & $39(5.52 \%)$ & 0.7646 & $12(7.69 \%)$ & $38(5.29 \%)$ & 0.3637 \\
\hline Malignancy & $9(5.35 \%)$ & $156(22.09 \%)$ & $\begin{array}{l}<0.0001 * \\
* *\end{array}$ & $13(8.33 \%)$ & $152(21.16 \%)$ & $0.0022 * *$ \\
\hline $\begin{array}{l}\text { Metastatic } \\
\text { solid tumor }\end{array}$ & $5(2.97 \%)$ & $89(12.60 \%)$ & $0.0014 * *$ & $5(3.20 \%)$ & $89(12.39 \%)$ & $0.0031 * *$ \\
\hline \multicolumn{7}{|l|}{ Medications } \\
\hline ACEI & $27(16.07 \%)$ & $50(7.08 \%)$ & $0.0016^{* *}$ & $20(12.82 \%)$ & $57(7.93 \%)$ & 0.1081 \\
\hline $\mathrm{ARB}$ & $9(5.35 \%)$ & $21(2.97 \%)$ & 0.2191 & $9(5.76 \%)$ & $21(2.92 \%)$ & 0.146 \\
\hline $\begin{array}{l}\text { Lipid-loweri } \\
\text { ng drugs }\end{array}$ & - & - & - & - & - & - \\
\hline $\begin{array}{l}\text { Anticoagulan } \\
\text { ts }\end{array}$ & $33(19.64 \%)$ & $67(9.49 \%)$ & $0.0020 * *$ & $27(17.30 \%)$ & $73(10.16 \%)$ & $0.0372 *$ \\
\hline Insulin & - & - & - & - & - & - \\
\hline $\begin{array}{l}\text { Incretin } \\
\text { mimetics }\end{array}$ & $4(2.38 \%)$ & $6(0.84 \%)$ & 0.2118 & $1(0.64 \%)$ & $9(1.25 \%)$ & 0.8183 \\
\hline \multicolumn{7}{|l|}{ Complete } \\
\hline \multicolumn{7}{|l|}{ blood counts } \\
\hline $\begin{array}{l}\text { Mean } \\
\text { corpuscular } \\
\text { volume, fL }\end{array}$ & $90.6(87.25-93.4) ; \mathrm{n}=122$ & $89.5(85.9-93.3) ; \mathrm{n}=421$ & 0.1331 & $91.0(87.3-93.8) ; \mathrm{n}=117$ & $89.45(85.8-93.05) ; \mathrm{n}=426$ & $0.0247 *$ \\
\hline $\begin{array}{l}\text { Eosinophil, } \\
\times 10^{\wedge} 9 / \mathrm{L}\end{array}$ & $0.1(0.0-0.26) ; \mathrm{n}=113$ & $0.1(0.0-0.2) ; \mathrm{n}=391$ & $0.0387^{*}$ & $0.1(0.0-0.2) ; \mathrm{n}=107$ & $0.1(0.0-0.2) ; \mathrm{n}=397$ & 0.633 \\
\hline
\end{tabular}




\begin{tabular}{|c|c|c|c|c|c|c|}
\hline $\begin{array}{l}\text { Lymphocyte, } \\
\text { x10^9/L }\end{array}$ & $1.1(0.8-1.6) ; \mathrm{n}=113$ & $1.2(0.72-1.7) ; \mathrm{n}=392$ & 0.9579 & $1.2(0.78-1.7) ; \mathrm{n}=108$ & $1.1(0.8-1.7) ; \mathrm{n}=397$ & 0.3458 \\
\hline $\begin{array}{l}\text { Monocyte, } \\
\times 10^{\wedge} 9 / \mathrm{L}\end{array}$ & $0.6(0.4-0.8) ; \mathrm{n}=113$ & $0.6(0.4-0.8) ; \mathrm{n}=392$ & 0.385 & $0.6(0.4-0.7) ; \mathrm{n}=108$ & $0.6(0.4-0.8) ; \mathrm{n}=397$ & 0.6578 \\
\hline $\begin{array}{l}\text { Neutrophil, } \\
\times 10^{\wedge} 9 / \mathrm{L}\end{array}$ & $5.4(3.9-8.6) ; \mathrm{n}=113$ & $4.8(3.3-7.45) ; \mathrm{n}=392$ & $0.0304 *$ & $4.9(3.35-7.9) ; \mathrm{n}=108$ & $4.9(3.4-7.6) ; \mathrm{n}=397$ & 0.7809 \\
\hline $\begin{array}{l}\text { White cell } \\
\text { count, } \\
\times 10^{\wedge} 9 / \mathrm{L}\end{array}$ & $7.45(6.0-10.3) ; \mathrm{n}=122$ & $6.9(5.5-8.8) ; \mathrm{n}=421$ & $0.0383 *$ & $6.9(5.6-9.2) ; \mathrm{n}=117$ & $7.05(5.5-9.05) ; \mathrm{n}=426$ & 0.9208 \\
\hline $\begin{array}{l}\text { Mean cell } \\
\text { haemoglobin, } \\
\text { pg }\end{array}$ & $30.85(29.4-32.3) ; \mathrm{n}=122$ & $30.6(28.9-32.0) ; n=421$ & 0.385 & $30.9(29.5-32.0) ; \mathrm{n}=117$ & $30.5(28.95-32.0) ; \mathrm{n}=426$ & 0.2106 \\
\hline $\begin{array}{l}\text { Platelet, } \\
\times 10^{\wedge} 9 / \mathrm{L}\end{array}$ & $196.5(151.5-259.5) ; \mathrm{n}=122$ & $218.0(173.0-280.0) ; \mathrm{n}=421$ & $0.0058 * *$ & $199.0(163.0-255.0) ; \mathrm{n}=117$ & $216.5(171.5-284.0) ; n=426$ & $0.022 *$ \\
\hline $\begin{array}{l}\text { Red blood } \\
\text { count, } \\
\times 10^{\wedge} 12 / \mathrm{L}\end{array}$ & $4.02(3.53-4.47) ; \mathrm{n}=122$ & $4.1(3.64-4.62) ; n=421$ & 0.1079 & $4.18(3.72-4.53) ; \mathrm{n}=117$ & $4.08(3.57-4.58) ; \mathrm{n}=426$ & 0.4458 \\
\hline $\begin{array}{l}\text { Liver and } \\
\text { renal } \\
\text { functions }\end{array}$ & & & & & & \\
\hline $\begin{array}{l}\mathrm{K} / \text { Potassium, } \\
\mathrm{mmol} / \mathrm{L}\end{array}$ & $4.1(3.64-4.4) ; n=149$ & $4.0(3.7-4.4) ; \mathrm{n}=535$ & 0.8865 & $4.1(3.8-4.4) ; \mathrm{n}=136$ & $4.0(3.7-4.4) ; \mathrm{n}=548$ & $0.0182 *$ \\
\hline $\begin{array}{l}\text { Urate, } \\
\mathrm{mmol} / \mathrm{L}\end{array}$ & $0.46(0.36-0.6) ; \mathrm{n}=27$ & $0.33(0.25-0.45) ; \mathrm{n}=73$ & $0.0011 * *$ & $0.44(0.34-0.55) ; \mathrm{n}=20$ & $0.34(0.26-0.47) ; \mathrm{n}=80$ & 0.0751 \\
\hline Albumin, $\mathrm{g} / \mathrm{L}$ & $35.9(31.4-38.5) ; \mathrm{n}=149$ & $35.9(30.25-40.2) ; \mathrm{n}=532$ & 0.6972 & $36.75(31.35-41.4) ; \mathrm{n}=134$ & $35.6(30.35-39.8) ; \mathrm{n}=547$ & 0.0835 \\
\hline $\mathrm{Na} /$ Sodium, & $139.2(135.7-141.7) ; \mathrm{n}=149$ & $138.3(135.1-140.6) ; \mathrm{n}=535$ & $0.0198 *$ & $139.55(137.2-142.0) ; \mathrm{n}=13$ & $138.2(134.8-140.7) ; n=548$ & $0.0023 * *$ \\
\hline
\end{tabular}


$\mathrm{mmol} / \mathrm{L}$

Urea, $\mathrm{mmol} / \mathrm{L}$

Protein, g/L 70.5(66.1-75.0);n=149

Creatinine, $\quad 98.0(75.5-145.0) ; \mathrm{n}=148$ umol/L

Alkaline

phosphatase, 84.0(67.0-125.0);n=149

$\mathrm{U} / \mathrm{L}$

\section{Aspartate}

transaminase, 26.0(18.0-42.9); $\mathrm{n}=25$

$\mathrm{U} / \mathrm{L}$

Alanine

transaminase, $24.0(16.0-40.0) ; \mathrm{n}=149$

$\mathrm{U} / \mathrm{L}$

Bilirubin,

umol/L

$13.3(7.4-19.1) ; \mathrm{n}=149$

Lipid and

glucose tests

Triglyceride, $\mathrm{mmol} / \mathrm{L}$

$0.96(0.76-1.23) ; \mathrm{n}=43$

Low-density

lipoprotein, $\quad 1.89(1.45-2.57) ; \mathrm{n}=41$

$\mathrm{mmol} / \mathrm{L}$

High-density

lipoprotein, $0.96(0.8-1.31) ; \mathrm{n}=41$

\begin{tabular}{|c|c|c|c|c|}
\hline $5.48(4.1-8.1) ; n=534$ & $\begin{array}{l}<0.0001 * \\
* *\end{array}$ & $6.5(4.98-8.43) ; n=136$ & $5.67(4.1-8.35) ; \mathrm{n}=547$ & $0.0037 * *$ \\
\hline $71.2(65.45-75.55) ; \mathrm{n}=532$ & 0.9483 & $71.35(67.3-75.7) ; \mathrm{n}=134$ & $71.0(65.1-75.35) ; \mathrm{n}=547$ & 0.2734 \\
\hline $80.0(63.0-105.5) ; \mathrm{n}=534$ & $\begin{array}{l}<0.0001 * \\
* *\end{array}$ & $97.0(73.5-119.5) ; \mathrm{n}=135$ & $80.0(63.0-110.0) ; \mathrm{n}=547$ & $0.0011 * *$ \\
\hline $81.0(64.0-117.0) ; \mathrm{n}=532$ & 0.2434 & $81.5(66.0-109.5) ; \mathrm{n}=134$ & $82.0(64.0-122.0) ; \mathrm{n}=547$ & 0.5168 \\
\hline $40.0(19.0-73.0) ; \mathrm{n}=57$ & 0.0915 & $22.5(12.5-43.0) ; \mathrm{n}=20$ & $38.5(20.55-62.5) ; \mathrm{n}=62$ & 0.1018 \\
\hline $24.0(16.0-45.0) ; \mathrm{n}=532$ & 0.777 & $23.0(16.0-35.0) ; \mathrm{n}=134$ & $25.0(16.0-47.5) ; \mathrm{n}=547$ & 0.0702 \\
\hline $9.95(6.4-16.0) ; \mathrm{n}=532$ & $\begin{array}{l}0.0007 * * \\
*\end{array}$ & $12.85(7.4-18.85) ; \mathrm{n}=134$ & $10.0(6.65-16.05) ; \mathrm{n}=547$ & $0.0133^{*}$ \\
\hline $1.02(0.74-1.6) ; \mathrm{n}=124$ & 0.1615 & $0.9(0.75-1.06) ; \mathrm{n}=28$ & $1.05(0.75-1.52) ; \mathrm{n}=139$ & $0.0387 *$ \\
\hline $2.32(1.69-3.03) ; \mathrm{n}=112$ & $0.0307 *$ & $1.69(1.42-2.54) ; \mathrm{n}=27$ & $2.24(1.66-3.03) ; \mathrm{n}=126$ & $0.0274^{*}$ \\
\hline $1.1(0.85-1.51) ; \mathrm{n}=112$ & 0.1375 & $1.2(0.79-1.4) ; \mathrm{n}=27$ & $1.06(0.82-1.44) ; \mathrm{n}=126$ & 0.9409 \\
\hline
\end{tabular}


$\mathrm{mmol} / \mathrm{L}$

Cholesterol,

$\mathrm{mmol} / \mathrm{L}$

$3.48(2.7-4.12) ; n=43$

$4.0(3.36-4.9) ; \mathrm{n}=124 \quad 0.007 * *$

$3.43(2.79-4.17) ; \mathrm{n}=28 \quad 3.9(3.36-4.86) ; \mathrm{n}=139 \quad 0.0173^{*}$

HbA1c, g/dL 12.3(10.5-13.5);n=122

$12.3(10.9-13.7) ; n=421$

0.3114

$12.6(11.3-13.7) ; \mathrm{n}=117$

$12.1(10.6-13.7) ; \mathrm{n}=426$

0.0736

Glucose,

$\mathrm{mmol} / \mathrm{L}$

$$
6.74(5.38-8.8) ; \mathrm{n}=104
$$

$6.35(5.46-7.98) ; \mathrm{n}=363$

0.1414

$6.73(5.44-8.76) ; \mathrm{n}=95$

$6.4(5.46-7.91) ; \mathrm{n}=372$

0.3817

Cardiac,

clotting,

\section{inflammator}

$y$,

and

\section{acid-base}

tests

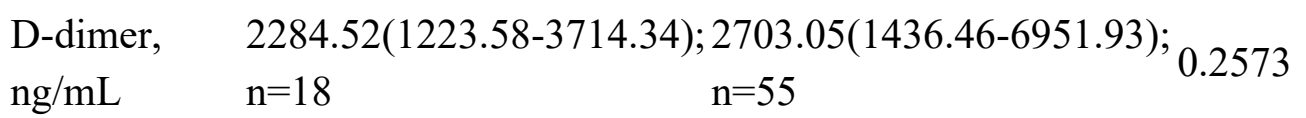

High

sensitive

troponin-I,

$0.04(0.02-0.13) ; \mathrm{n}=113$

$0.02(0.01-0.05) ; \mathrm{n}=331$

$\mathrm{ng} / \mathrm{L}$

Lactate

dehydrogena 256.0(217.0-335.0);n=119 267.5(207.0-399.0);n=364 0.4864 se, U/L

APTT,

second

$34.6(30.55-39.35) ; \mathrm{n}=155 \quad 32.8(30.1-35.9) ; \mathrm{n}=566$

Prothrombin time/INR,

\footnotetext{
$11.9(10.8-14.05) ; \mathrm{n}=155 \quad 11.9(10.8-13.65) ; \mathrm{n}=566$
}

0.3178

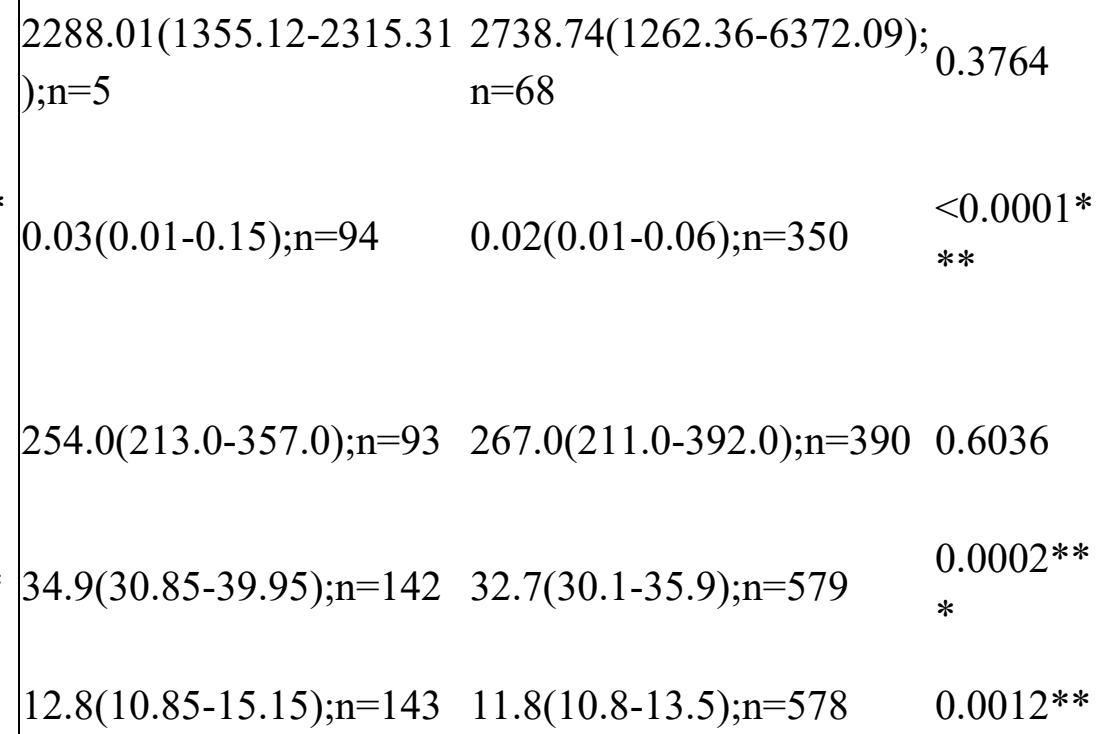

$0.0016^{* *} \quad 34.9(30.85-39.95) ; \mathrm{n}=142 \quad 32.7(30.1-35.9) ; \mathrm{n}=579$

$0.0002 * *$ 
second

C-reactive

protein,

$1.94(0.38-7.46) ; \mathrm{n}=136 \quad 3.25(0.64-8.99) ; \mathrm{n}=420$

0.1109

$2.27(0.39-9.39) ; \mathrm{n}=115 \quad 3.05(0.73-8.33) ; \mathrm{n}=441$

0.5787

$\mathrm{mg} / \mathrm{dL}$

ECG

measuremen

ts

Trans QRS

initangle

Trans QRS

initmag

$0.38(0.25-0.63) ; \mathrm{n}=110 \quad 0.29(0.18-0.46) ; \mathrm{n}=464$

Trans QRS

max angle

Trans QRS

max mag

$324.0(234.0-342.0) ; \mathrm{n}=110 \quad 332.0(26.5-348.0) ; \mathrm{n}=464$

$1.33(0.97-2.03) ; \mathrm{n}=110 \quad 1.2(0.83-1.62) ; \mathrm{n}=464$

Trans QRS

term angle

$237.5(214.0-268.0) ; \mathrm{n}=110 \quad 244.5(214.5-273.5) ; \mathrm{n}=464$

Trans QRS

term mag

$0.36(0.25-0.5) ; \mathrm{n}=110 \quad 0.28(0.18-0.39) ; \mathrm{n}=464 \quad<0.0001^{*}$

Trans QRS $\quad-100.0(-100.0--36.0) ; \mathrm{n}=11 \quad-100.0(-100.0--100.0) ; \mathrm{n}=4$

cwrot 0

64

0.107

$0.32(0.2-0.48) ; \mathrm{n}=96$

$\mathrm{P}$ wave front

axis

$56.0(34.5-69.0) ; \mathrm{n}=136$

$54.0(39.0-68.0) ; \mathrm{n}=662$

0.8712

$-100.0(-100.0--34.5) ; \mathrm{n}=96_{78}^{-100 .}$

$100.0(-100.0--100.0) ; \mathrm{n}=4$

$$
78
$$

$54.0(35.0-70.0) ; \mathrm{n}=113 \quad 55.0(39.0-68.0) ; \mathrm{n}=685$

0.8949

P wave

horizon axis

$16.0(-5.0-36.5) ; \mathrm{n}=155$

$21.0(5.0-37.0) ; \mathrm{n}=675$

0.0769

$16.0(-3.5-49.5) ; \mathrm{n}=135$

$21.0(5.0-36.0) ; \mathrm{n}=695$

0.91

I 40 front

$34.5(2.0-55.0) ; \mathrm{n}=166$

$39.0(18.0-58.0) ; \mathrm{n}=694$

$0.0252 * 36.5(14.5-58.0) ; \mathrm{n}=154 \quad 38.0(15.0-57.5) ; \mathrm{n}=706$

0.6716 
axis

I 40 horizon

axis

$26.0(11.0-43.0) ; \mathrm{n}=161$

$28.0(12.0-44.0) ; \mathrm{n}=678$

0.5733

$23.0(10.5-38.0) ; \mathrm{n}=150$

$29.0(13.0-45.0) ; \mathrm{n}=689$

0.0164*

QRS front

axis

$41.0(0.5-77.5) ; \mathrm{n}=166$

$55.0(23.0-74.0) ; \mathrm{n}=705$

$0.0093 * * 47.0(8.0-76.5) ; \mathrm{n}=155$

$54.5(22.0-74.0) ; \mathrm{n}=716$

0.0621

QRS horizon

axis

$-12.5(-33.0-10.0) ; n=164$

$-11.0(-30.0-8.0) ; \mathrm{n}=689$

0.6725

$-12.0(-34.0-6.5) ; \mathrm{n}=152$

$-11.0(-29.0-10.0) ; \mathrm{n}=701$

0.4055

T wave $40 \mathrm{~ms}$

front axis

$62.5(-10.0-105.0) ; n=164$

$69.5(23.0-100.0) ; \mathrm{n}=698$

0.2524

$69.0(7.0-101.0) ; \mathrm{n}=153$

$69.0(20.0-102.0) \cdot n=709$

0.6981

T wave $40 \mathrm{~ms}$

horizon axis

$-21.0(-56.0-222.0) ; \mathrm{n}=158$

$-23.0(-51.0-157.0) ; \mathrm{n}=671$

0.3101

$-20.0(-55.5-229.5) ; \mathrm{n}=144 \quad-23.0(-51.0-163.0) ; \mathrm{n}=685$

0.4783

ST segment

front axis

$83.0(22.0-168.0) ; \mathrm{n}=153$

$61.0(30.5-101.0) ; \mathrm{n}=674$

$0.0223 *$

ST segment

horizon axis

$99.0(65.0-161.0) ; \mathrm{n}=143$

$71.0(48.0-107.0) ; \mathrm{n}=637$

$<0.0001 *$

$\mathrm{T}$ wave front

axis

$52.0(10.0-105.0) ; \mathrm{n}=161$

$44.0(14.5-68.5) ; \mathrm{n}=696$

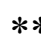

$76.5(38.5-162.5) ; \mathrm{n}=144$

$62.0(29.0-105.0) ; \mathrm{n}=683$

0.0207*

96.5

$<0.0001^{*}$

**

$0.0378^{*} \quad 43.0(11.5-72.5) ; \mathrm{n}=150 \quad 45.0(15.0-71.0) ; \mathrm{n}=707 \quad 0.5477$

T wave

horizon axis

$63.0(26.0-108.5) ; \mathrm{n}=155$

$44.0(25.0-71.0) ; \mathrm{n}=650$

$0.0014 * *$

$46.5(25.0-79.5) ; n=142$

Atrialrate

$81.0(67.0-102.5) ; \mathrm{n}=168$

$80.0(66.0-99.0) ; \mathrm{n}=706$

0.2339

$79.0(64.0-102.5) ; \mathrm{n}=156$

$46.0(24.5-75.0) ; n=663$

0.6236

Mean

ventricular

$80.5(67.5-94.0) ; \mathrm{n}=168$

$81.0(68.0-98.0) ; \mathrm{n}=706$

0.4043

$77.5(67.5-89.0) ; n=156$

$80.5(67.0-99.0) ; \mathrm{n}=718$

0.8544

rate

Mean PT interval $166.0(152.0-186.0) ; \mathrm{n}=168 \quad 160.0(144.0-176.0) ; \mathrm{n}=706$

$0.0001 * *$

$172.0(154.0-192.0) ; \mathrm{n}=156$ 160.0(144.0-176.0);n=718 $* *$ Mean PR $68.0(53.0-86.5) ; \mathrm{n}=168$ $62.0(48.0-75.0) ; \mathrm{n}=706$

$61.0(48.0-74.5) ; \mathrm{n}=718$

$<0.0001^{*}$ 
segment

Mean QRS

duration

$90.0(80.0-101.5) ; \mathrm{n}=168 \quad 84.0(78.0-94.0) ; \mathrm{n}=706$

Mean QT

interval

$395.5(362.5-422.0) ; \mathrm{n}=168 \quad 374.0(343.0-408.0) ; \mathrm{n}=706$

Mean QTc $\quad 447.0(423.5-474.5) ; \mathrm{n}=168 \quad 431.0(410.0-456.0) ; \mathrm{n}=706$

QT interval

dispersion

$77.0(0.0-119.0) ; \mathrm{n}=168 \quad 63.0(0.0-101.5) ; \mathrm{n}=706$

$<0.0001 *$

$88.0(81.5-96.0) ; \mathrm{n}=156 \quad 84.0(78.0-94.5) ; \mathrm{n}=718 \quad 0.0059^{* *}$

$<0.0001 *$

$<0.0001^{*}$

$* *$

$443.5(420.5-463.0) ; \mathrm{n}=156432.0(411.0-457.0) ; \mathrm{n}=718 \quad 0.0022^{* *}$

COPD: chronic obstructive pulmonary disease; ACEI: angiotensinogen converting enzyme inhibitor; ARB: angiotensin receptor blocker; APTT: activated partial thromboplastin time. 
Table 3. Univariate Cox analysis of significant risk factors to predict spontaneous VT/VF, new onset HF and new onset AF.

$*$ for $\mathrm{p} \leq 0.05, * *$ for $\mathrm{p} \leq 0.01, * * *$ for $\mathrm{p} \leq 0.001$

\begin{tabular}{|c|c|c|c|c|c|c|}
\hline Characteristics & $\begin{array}{l}\text { Spontaneous VT/VF } \\
\text { HR }[95 \% \text { CI];P value }\end{array}$ & Cutoff & $\begin{array}{l}\text { New onset HF } \\
\text { HR }[95 \% \text { CI];P value }\end{array}$ & Cutoff & $\begin{array}{l}\text { New onset AF } \\
\text { HR }[95 \% \text { CI];P value }\end{array}$ & Cutoff \\
\hline \multicolumn{7}{|l|}{ Demographics } \\
\hline Male gender & $0.84[0.50-1.42] ; 0.5177$ & - & $0.91[0.67-1.25] ; 0.5643$ & - & $0.95[0.68-1.31] ; 0.7399$ & - \\
\hline Female gender & $1.19[0.70-2.01] ; 0.5177$ & - & $1.10[0.80-1.50] ; 0.5643$ & - & $1.06[0.76-1.46] ; 0.7399$ & - \\
\hline Baseline age, years & $1.00[0.98-1.01] ; 0.6879$ & 35.13 & $1.04[1.03-1.05] ;<0.0001 * * *$ & 77.11 & $1.04[1.03-1.06] ;<0.0001 * * *$ & 49.46 \\
\hline$<40$ & $1.55[0.82-2.93] ; 0.1808$ & - & $0.35[0.18-0.66] ; 0.0013 * *$ & - & $0.07[0.02-0.27] ; 0.0001 * * *$ & - \\
\hline$[40,50)$ & $0.64[0.26-1.60] ; 0.3409$ & - & $0.42[0.22-0.80] ; 0.0085^{* *}$ & - & $0.29[0.14-0.63] ; 0.0016^{* *}$ & - \\
\hline$[50-60)$ & $0.86[0.46-1.61] ; 0.6421$ & - & $0.58[0.38-0.87] ; 0.0094 * *$ & - & $0.98[0.68-1.42] ; 0.9185$ & - \\
\hline$[60-70)$ & $1.09[0.59-1.99] ; 0.7835$ & - & $1.45[1.03-2.03] ; 0.0328^{*}$ & - & $1.74[1.24-2.45] ; 0.0015^{* *}$ & - \\
\hline$[70-80)$ & $1.19[0.60-2.36] ; 0.6219$ & - & $1.62[1.11-2.35] ; 0.0121^{*}$ & - & $1.87[1.28-2.74] ; 0.0013^{* *}$ & - \\
\hline$>=80$ & $0.50[0.12-2.06] ; 0.3382$ & - & $3.40[2.27-5.10] ;<0.0001 * * *$ & - & $1.93[1.18-3.16] ; 0.0093^{* *}$ & - \\
\hline \multicolumn{7}{|l|}{ Past comorbidities } \\
\hline Baseline VT/VF & $13.06[4.70-36.28] ;<0.0001 * * *$ & & $2.12[0.68-6.65] ; 0.1967$ & - & $0.66[0.09-4.70] ; 0.6754$ & - \\
\hline $\begin{array}{l}\text { Cerebrovascular } \\
\text { disease }\end{array}$ & $1.12[0.27-4.58] ; 0.8790$ & - & $1.96[1.00-3.84] ; 0.0504$ & - & $1.59[0.74-3.39] ; 0.2346$ & - \\
\hline Rheumatic disease & $2.21[0.80-6.14] ; 0.1269$ & - & $0.84[0.31-2.26] ; 0.7284$ & - & $0.56[0.18-1.75] ; 0.3153$ & - \\
\hline Renal diseases & $0.88[0.28-2.83] ; 0.8364$ & - & $1.55[0.90-2.69] ; 0.1158$ & - & $1.17[0.63-2.17] ; 0.6117$ & - \\
\hline Diabetes mellitus & $1.99[0.62-6.37] ; 0.2471$ & - & $2.55[1.34-4.83] ; 0.0043^{* *}$ & - & $1.50[0.66-3.40] ; 0.3321$ & - \\
\hline Hypertension & $1.21[0.62-2.34] ; 0.5752$ & - & $2.73[1.96-3.80] ;<0.0001 * * *$ & - & $1.61[1.10-2.35] ; 0.0149^{*}$ & - \\
\hline Malignant dysrhythmia & $9.05[3.60-22.70] ;<0.0001 * * *$ & - & $1.65[0.61-4.44] ; 0.3254$ & - & $1.36[0.43-4.27] ; 0.5977$ & - \\
\hline AMI & - & - & $2.51[1.18-5.36] ; 0.0174 *$ & - & $1.19[0.44-3.23] ; 0.7280$ & - \\
\hline COPD & $2.10[0.29-15.19] ; 0.4622$ & - & $2.27[0.72-7.12] ; 0.1600$ & - & $0.72[0.10-5.13] ; 0.7410$ & - \\
\hline
\end{tabular}




\begin{tabular}{|c|c|c|c|}
\hline IHD & $1.04[0.45-2.42] ; 0.9293$ & - & $2.04[1.35-3.08] ; 0.0008^{* * *}$ \\
\hline PVD & $1.20[0.17-8.69] ; 0.8557$ & - & $1.67[0.62-4.50] ; 0.3135$ \\
\hline Stroke/TIA & $1.22[0.30-5.02] ; 0.7803$ & - & $1.64[0.77-3.51] ; 0.1988$ \\
\hline $\begin{array}{l}\text { Gastrointestinal } \\
\text { bleeding }\end{array}$ & $0.90[0.22-3.68] ; 0.8804$ & - & $1.58[0.83-2.99] ; 0.1628$ \\
\hline $\begin{array}{l}\text { Immune mediated } \\
\text { enterocolitis }\end{array}$ & $0.62[0.15-2.54] ; 0.5065$ & - & $0.74[0.35-1.58] ; 0.4382$ \\
\hline $\begin{array}{l}\text { Anxiety disorder and } \\
\text { depression }\end{array}$ & $0.94[0.13-6.77] ; 0.9478$ & - & $2.73[1.28-5.83] ; 0.0095 * *$ \\
\hline SCD & $0.82[0.11-5.92] ; 0.8431$ & - & $2.26[1.06-4.82] ; 0.0355^{*}$ \\
\hline Atrial flutter & - & - & $1.07[0.15-7.62] ; 0.9485$ \\
\hline Accident fall & $1.03[0.32-3.30] ; 0.9617$ & - & $1.25[0.67-2.30] ; 0.4828$ \\
\hline Malignancy & $1.04[0.49-2.21] ; 0.9208$ & - & $0.32[0.16-0.63] ; 0.0010 * *$ \\
\hline Metastatic solid tumor & $0.63[0.15-2.62] ; 0.5274$ & - & $0.46[0.19-1.12] ; 0.0873$ \\
\hline \multicolumn{4}{|l|}{ Medications } \\
\hline ACEI & $1.27[0.58-2.81] ; 0.5525$ & - & $1.98[1.31-2.99] ; 0.0013 * *$ \\
\hline $\mathrm{ARB}$ & $2.13[0.85-5.35] ; 0.1074$ & - & $1.33[0.68-2.60] ; 0.4116$ \\
\hline Lipid-lowering drugs & - & - & - \\
\hline Anticoagulants & $1.57[0.81-3.05] ; 0.1776$ & - & $1.80[1.23-2.65] ; 0.0026^{* *}$ \\
\hline Insulin & - & - & - \\
\hline Incretin mimetics & $1.31[0.18-9.50] ; 0.7869$ & - & $2.26[0.84-6.11] ; 0.1069$ \\
\hline \multicolumn{4}{|l|}{ Complete blood counts } \\
\hline $\begin{array}{l}\text { Mean corpuscular } \\
\text { volume, } \mathrm{fL}\end{array}$ & $0.98[0.94-1.01] ; 0.2003$ & 90 & $1.02[0.99-1.04] ; 0.1297$ \\
\hline Eosinophil, $\times 10^{\wedge} 9 / \mathrm{L}$ & $1.52[0.81-2.86] ; 0.1902$ & 0.17 & $1.38[0.97-1.97] ; 0.0773$ \\
\hline
\end{tabular}

\begin{tabular}{|c|c|}
\hline $.49[0.94-2.36] ; 0.0935$ & - \\
\hline $41[0.45-4.43] ; 0.5563$ & - \\
\hline $78[0.83-3.81] ; 0.1352$ & - \\
\hline $59[0.81-3.11] ; 0.1801$ & - \\
\hline $70[0.31-1.58] ; 0.3893$ & - \\
\hline $74[0.18-3.01] ; 0.6789$ & - \\
\hline $10[0.41-2.98] ; 0.8475$ & - \\
\hline $61[2.07-15.20] ; 0.0007 * * *$ & - \\
\hline $71[0.95-3.08] ; 0.0761$ & - \\
\hline $50[0.27-0.90] ; 0.0202^{*}$ & - \\
\hline $52[0.21-1.28] ; 0.1530$ & - \\
\hline $30[0.80-2.11] ; 0.2826$ & - \\
\hline $45[0.74-2.85] ; 0.2802$ & - \\
\hline & - \\
\hline $38[0.90-2.11] ; 0.1355$ & - \\
\hline & - \\
\hline $47[0.07-3.33] ; 0.4469$ & - \\
\hline $2[0.99-1.04] ; 0.1925$ & 90.7 \\
\hline$[0.53-1.75] ; 0.9094$ & 0.09 \\
\hline
\end{tabular}


Lymphocyte, $x 10^{\wedge} 9 / \mathrm{L} \quad 0.82[0.50-1.34] ; 0.421$

Monocyte, $x 10^{\wedge} 9 / \mathrm{L} \quad 1.37[0.94-1.99] ; 0.1023$

Neutrophil, x10^9/L $\quad 0.98[0.89-1.08] ; 0.7162$

White cell count,

$\mathrm{x} 10^{\wedge} 9 / \mathrm{L}$

Mean cell

haemoglobin, pg

Platelet, $\mathrm{x} 10^{\wedge} 9 / \mathrm{L}$

Red blood count,

$\mathrm{x} 10^{\wedge} 12 / \mathrm{L}$

\section{Liver and renal}

\section{functions}

$\mathrm{K} /$ Potassium, mmol/L

Urate, $\mathrm{mmol} / \mathrm{L}$

Albumin, g/L

$1.07[0.99-1.16] ; 0.0963$

$0.94[0.86-1.03] ; 0.1745$

$1.002[0.998-1.005] ; 0.3552$

$0.83[0.55-1.26] ; 0.3890$

$\mathrm{Na} /$ Sodium, $\mathrm{mmol} / \mathrm{L}$

Urea, $\mathrm{mmol} / \mathrm{L}$

Protein, g/L

Creatinine, umol/L

Alkaline phosphatase,

$\mathrm{U} / \mathrm{L}$

Aspartate transaminase

$\mathrm{U} / \mathrm{L}$

$0.90[0.57-1.43] ; 0.6644$

$1.92[0.07-50.43] ; 0.6952$

$0.98[0.94-1.01] ; 0.2225$

$0.99[0.93-1.05] ; 0.7526$

$1.01[0.97-1.04] ; 0.7378$

$0.99[0.96-1.02] ; 0.5079$

$1.000[0.999-1.001] ; 0.6363$

$1.00[1.00-1.01] ; 0.3039$

$1.001[0.999-1.002] ; 0.2601$

Alanine transaminase,

$\mathrm{U} / \mathrm{L}$

\begin{tabular}{|c|c|c|c|c|}
\hline 1.1 & $0.90[0.70-1.15] ; 0.3919$ & 1.15 & $0.99[0.78-1.25] ; 0.9186$ & 0.6 \\
\hline 0.65 & $1.40[1.13-1.75] ; 0.0025^{* *}$ & 0.49 & $1.03[0.72-1.49] ; 0.8588$ & 0.47 \\
\hline 4.9 & $1.06[1.01-1.11] ; 0.0084 * *$ & 4.7 & $1.04[0.99-1.09] ; 0.1068$ & 6.3 \\
\hline 6.4 & $1.07[1.03-1.12] ; 0.0017 * *$ & 8.3 & $1.01[0.96-1.07] ; 0.7197$ & 8.2 \\
\hline 30.6 & $1.02[0.96-1.08] ; 0.4921$ & 33.4 & $1.02[0.96-1.08] ; 0.5375$ & 30.4 \\
\hline 155 & $0.997[0.995-0.999] ; 0.0135^{*}$ & 215 & $0.998[0.996-1.000] ; 0.0943$ & 275 \\
\hline 3.4 & $0.76[0.61-0.95] ; 0.0180^{*}$ & 3.97 & $1.04[0.82-1.32] ; 0.7469$ & 3.62 \\
\hline 3.5 & $0.99[0.76-1.30] ; 0.9640$ & 3.64 & $1.27[0.98-1.64] ; 0.0716$ & 3.8 \\
\hline 0.57 & $34.00[3.86-299.69] ; 0.0015^{* *}$ & 0.43 & $3.17[0.25-40.88] ; 0.3769$ & 0.41 \\
\hline 39.8 & $0.98[0.96-1.00] ; 0.1147$ & 39.7 & $1.00[0.97-1.02] ; 0.8670$ & 25.3 \\
\hline 141.2 & $1.02[0.98-1.06] ; 0.3282$ & 141.1 & $1.05[1.00-1.09] ; 0.0366^{*}$ & 141.8 \\
\hline 11.89 & $1.02[1.01-1.03] ; 0.0053 * *$ & 6.6 & $1.01[0.99-1.03] ; 0.4214$ & 4.11 \\
\hline 77.2 & $0.99[0.97-1.01] ; 0.5958$ & 77.6 & $1.00[0.98-1.02] ; 0.9728$ & 66.4 \\
\hline 60 & $1.000[1.000-1.001] ; 0.5454$ & 122 & $1.000[0.999-1.001] ; 0.9813$ & 85 \\
\hline 67 & $1.001[0.999-1.004] ; 0.3772$ & 65 & $1.000[0.997-1.003] ; 0.8691$ & 63 \\
\hline 21 & $0.99[0.97-1.00] ; 0.1255$ & 49 & $1.000[0.998-1.001] ; 0.6384$ & 23 \\
\hline 15.9 & $1.001[1.000-1.001] ; 0.0027^{* *}$ & 91 & $1.00[0.99-1.00] ; 0.2503$ & 49 \\
\hline
\end{tabular}




\begin{tabular}{|c|c|c|c|c|c|c|}
\hline Bilirubin, umol/L & $0.99[0.97-1.02] ; 0.6014$ & 7.8 & $1.01[1.00-1.01] ; 0.0002 * * *$ & 12.3 & $1.01[1.01-1.02] ;<0.0001 * * *$ & 12 \\
\hline \multicolumn{7}{|l|}{ Lipid and glucose tests } \\
\hline Triglyceride, $\mathrm{mmol} / \mathrm{L}$ & $1.76[0.84-3.68] ; 0.1323$ & 1.21 & $0.61[0.34-1.11] ; 0.1053$ & 1.69 & $0.37[0.15-0.90] ; 0.0281^{*}$ & 1.23 \\
\hline $\begin{array}{l}\text { Low-density } \\
\text { lipoprotein, } \mathrm{mmol} / \mathrm{L}\end{array}$ & $0.97[0.54-1.74] ; 0.9228$ & 1.2109 & $0.66[0.45-0.95] ; 0.0273 *$ & 2.1173 & $0.65[0.40-1.04] ; 0.0724$ & 2.7855 \\
\hline $\begin{array}{l}\text { High-density } \\
\text { lipoprotein, mmol/L }\end{array}$ & $0.19[0.04-0.94] ; 0.0415^{*}$ & 1.09 & $0.56[0.28-1.14] ; 0.1109$ & 1.56 & $0.96[0.42-2.19] ; 0.9242$ & 1.18 \\
\hline Cholesterol, mmol/L & $0.99[0.65-1.52] ; 0.9722$ & 4.03 & $0.70[0.53-0.93] ; 0.0126^{*}$ & 4.7 & $0.68[0.47-0.97] ; 0.0343^{*}$ & 3.6 \\
\hline $\mathrm{HbA1c}, \mathrm{g} / \mathrm{dL}$ & $0.90[0.78-1.04] ; 0.1583$ & 11.4 & $0.92[0.85-1.00] ; 0.0464 *$ & 9.7 & $1.03[0.95-1.13] ; 0.4444$ & 10.8 \\
\hline Glucose, $\mathrm{mmol} / \mathrm{L}$ & $0.96[0.86-1.07] ; 0.4460$ & 5.17 & $1.05[1.01-1.09] ; 0.0165^{*}$ & 6.64 & $1.03[0.98-1.07] ; 0.2317$ & 7.79 \\
\hline \multicolumn{7}{|l|}{$\begin{array}{l}\text { Cardiac, clotting, } \\
\text { inflammatory, } \\
\text { and acid-base tests }\end{array}$} \\
\hline D-dimer, ng/mL & $1.000[1.000-1.000] ; 0.8538$ & 1676.12 & $1.000[1.000-1.000] ; 0.2234$ & 6329.5498 & $1.000[1.000-1.000] ; 0.9549$ & 2315.31 \\
\hline $\begin{array}{l}\text { High sensitive } \\
\text { troponin-I, ng/L }\end{array}$ & $0.99[0.90-1.08] ; 0.7459$ & 0.0199 & $1.01[1.00-1.02] ; 0.1592$ & 0.0179 & $1.00[0.98-1.02] ; 0.9476$ & 0.0114 \\
\hline $\begin{array}{l}\text { Lactate dehydrogenase, } \\
\mathrm{U} / \mathrm{L}\end{array}$ & $1.001[1.000-1.002] ; 0.0554$ & 409 & $1.000[0.999-1.001] ; 0.8341$ & 218 & $1.001[1.000-1.001] ; 0.1068$ & 223 \\
\hline APTT, second & $1.01[0.99-1.03] ; 0.3782$ & 33.9 & $1.01[1.00-1.02] ; 0.0073^{* *}$ & 39.5 & $1.02[1.01-1.03] ;<0.0001 * * *$ & 39.5 \\
\hline $\begin{array}{l}\text { Prothrombin time/INR, } \\
\text { second }\end{array}$ & $1.00[0.96-1.05] ; 0.8719$ & 10.1 & $1.03[1.01-1.05] ; 0.0030 * *$ & 16.1 & $1.04[1.02-1.06] ; 0.0002 * * *$ & 12.7 \\
\hline $\begin{array}{l}\text { C-reactive protein, } \\
\mathrm{mg} / \mathrm{dL}\end{array}$ & $1.01[0.97-1.05] ; 0.5465$ & 1.739 & $1.00[0.97-1.02] ; 0.8999$ & 4.175 & $1.01[0.99-1.03] ; 0.4155$ & 5.975 \\
\hline \multicolumn{7}{|l|}{ ECG measurements } \\
\hline Trans QRS initangle & $1.000[0.997-1.003] ; 0.8486$ & 21 & $1.002[1.001-1.004] ; 0.0037 * *$ & 205 & $1.003[1.001-1.004] ; 0.0002 * * *$ & 251 \\
\hline Trans QRS initmag & $0.66[0.18-2.40] ; 0.5259$ & 0.11 & $2.77[1.67-4.60] ; 0.0001 * * *$ & 0.75 & $2.03[1.09-3.77] ; 0.0257^{*}$ & 0.582 \\
\hline
\end{tabular}




\begin{tabular}{|c|c|c|c|c|c|c|}
\hline Trans QRS max angle & $1.000[0.997-1.002] ; 0.7373$ & 220 & $1.001[0.999-1.002] ; 0.3282$ & 351 & $\mid 1.001[1.000-1.003] ; 0.0752$ & 7 \\
\hline Trans QRS max mag & $0.70[0.39-1.24] ; 0.2230$ & 1.322 & $1.43[1.08-1.90] ; 0.0131^{*}$ & 2.16 & $1.23[0.91-1.67] ; 0.1847$ & 1.97 \\
\hline Trans QRS term angle & $1.00[0.99-1.00] ; 0.0444 *$ & 234 & $1.00[0.99-1.00] ; 0.1175$ & 225 & $0.999[0.996-1.002] ; 0.5706$ & 257 \\
\hline Trans QRS term mag & $1.74[0.55-5.46] ; 0.3432$ & 0.592 & $2.70[1.55-4.68] ; 0.0004 * * *$ & 0.49 & $1.58[0.76-3.30] ; 0.2220$ & 0.45 \\
\hline Trans QRS cwrot & $1.00[1.00-1.01] ; 0.5411$ & -34 & $1.00[1.00-1.01] ; 0.2545$ & -50 & $1.00[1.00-1.01] ; 0.4167$ & -45 \\
\hline $\mathrm{P}$ wave front axis & $1.00[0.99-1.01] ; 0.7611$ & 75 & $1.00[1.00-1.01] ; 0.6109$ & 15 & $1.00[1.00-1.01] ; 0.2615$ & 75 \\
\hline $\mathrm{P}$ wave horizon axis & $1.00[0.99-1.00] ; 0.2539$ & -18 & $1.00[1.00-1.01] ; 0.2775$ & -15 & $1.01[1.00-1.01] ; 0.0002 * * *$ & 53 \\
\hline I 40 front axis & $1.00[0.99-1.00] ; 0.3288$ & -4 & $0.99[0.99-1.00] ; 0.0242 *$ & 7 & $1.00[0.99-1.00] ; 0.1402$ & 26 \\
\hline I 40 horizon axis & $1.01[1.00-1.01] ; 0.0587$ & -3 & $1.00[1.00-1.01] ; 0.2961$ & 29 & $0.99[0.99-1.00] ; 0.0172 *$ & 29 \\
\hline QRS front axis & $1.00[0.99-1.00] ; 0.5589$ & -12 & $0.99[0.99-1.00] ; 0.0017 * *$ & 2 & $1.00[0.99-1.00] ; 0.0128^{*}$ & -2 \\
\hline QRS horizon axis & $1.00[1.00-1.01] ; 0.7183$ & -48 & $1.002[0.999-1.004] ; 0.2684$ & -50 & $0.999[0.995-1.002] ; 0.4450$ & -30 \\
\hline $\mathrm{T}$ wave $40 \mathrm{~ms}$ front axis & $1.001[0.998-1.005] ; 0.4056$ & 103 & $0.999[0.996-1.001] ; 0.2277$ & -8 & $0.999[0.997-1.002] ; 0.5548$ & -41 \\
\hline $\begin{array}{l}\mathrm{T} \text { wave } 40 \mathrm{~ms} \text { horizon } \\
\text { axis }\end{array}$ & $1.002[1.000-1.004] ; 0.1059$ & 15 & $1.002[1.000-1.003] ; 0.0131 *$ & 91 & $1.001[1.000-1.002] ; 0.0744$ & 226 \\
\hline ST segment front axis & $1.001[0.997-1.004] ; 0.7597$ & 105 & $1.002[1.000-1.004] ; 0.0214^{*}$ & 108 & $1.00[1.00-1.01] ; 0.0011^{* *}$ & 106 \\
\hline $\begin{array}{l}\text { ST segment horizon } \\
\text { axis }\end{array}$ & $1.00[1.00-1.01] ; 0.2457$ & 149 & $1.01[1.00-1.01] ;<0.0001 * * *$ & 162 & $1.01[1.00-1.01] ; 0.0001 * * *$ & 90 \\
\hline $\mathrm{T}$ wave front axis & $1.00[1.00-1.01] ; 0.1033$ & 22 & $1.00[1.00-1.01] ; 0.0032 * *$ & 80 & $1.000[0.997-1.002] ; 0.8900$ & 28 \\
\hline T wave horizon axis & $1.000[0.996-1.005] ; 0.8465$ & 16 & $1.00[1.00-1.01] ; 0.0208^{*}$ & 59 & $1.000[0.997-1.003] ; 0.8419$ & 70 \\
\hline Atrialrate & $1.00[0.99-1.00] ; 0.4626$ & 71 & $1.00[1.00-1.01] ;<0.0001 * * *$ & 116 & $1.01[1.00-1.01] ;<0.0001 * * *$ & 118 \\
\hline Mean ventricular rate & $1.01[1.00-1.02] ; 0.1893$ & 77 & $1.00[1.00-1.01] ; 0.4141$ & 78 & $1.00[0.99-1.01] ; 0.9446$ & 103 \\
\hline Mean PT interval & $1.00[0.99-1.01] ; 0.8872$ & 162 & $1.01[1.00-1.01] ; 0.0006^{* * *}$ & 195 & $1.01[1.01-1.02] ;<0.0001 * * *$ & 176 \\
\hline Mean PR segment & $1.00[0.98-1.01] ; 0.4562$ & 56 & $1.01[1.00-1.01] ; 0.0046^{* *}$ & 90 & $1.01[1.01-1.02] ;<0.0001 * * *$ & 76 \\
\hline Mean QRS duration & $1.01[1.00-1.03] ; 0.0171^{*}$ & 93 & $1.02[1.01-1.02] ;<0.0001 * * *$ & 103 & $1.01[1.00-1.01] ; 0.0666$ & 77 \\
\hline Mean QT interval & $1.00[0.99-1.01] ; 0.9214$ & 338 & $1.01[1.00-1.01] ; 0.0011^{* *}$ & 391 & $1.00[1.00-1.01] ; 0.0051^{* *}$ & 392 \\
\hline
\end{tabular}


Mean QTc $\quad 1.01[1.00-1.02] ; 0.0035 * * \quad 413 \quad|1.01[1.01-1.02] ;<0.0001 * * * 466 \quad| 1.01[1.00-1.01] ; 0.0001 * * * \quad 445$

QT interval dispersion $1.001[0.997-1.005] ; 0.7497 \quad 34 \quad 1.00[1.00-1.01] ; 0.0004 * * * \quad 77 \quad 1.002[1.000-1.005] ; 0.0868 \quad 43$

COPD: chronic obstructive pulmonary disease; ACEI: angiotensinogen converting enzyme inhibitor; ARB: angiotensin receptor blocker; APTT: activated partial thromboplastin time.

Table 4. Univariate Cox analysis of significant risk factors to predict cardiovascular mortality and all-cause mortality.

$*$ for $\mathrm{p} \leq 0.05, * *$ for $\mathrm{p} \leq 0.01, * * *$ for $\mathrm{p} \leq 0.001$

\begin{tabular}{|c|c|c|c|c|}
\hline Characteristics & $\begin{array}{l}\text { Cardiovascularmortality } \\
\text { HR }[95 \% \text { CI }] ; P \text { value }\end{array}$ & Cutoff & $\begin{array}{l}\text { Mortality } \\
\text { HR [95\% CI];P value }\end{array}$ & Cutoff \\
\hline \multicolumn{5}{|l|}{ Demographics } \\
\hline Male gender & $0.88[0.50-1.55] ; 0.6610$ & - & $0.86[0.70-1.05] ; 0.1319$ & - \\
\hline Female gender & $1.13[0.65-1.99] ; 0.6610$ & - & $1.16[0.96-1.42] ; 0.1319$ & - \\
\hline Baseline age, years & $1.04[1.02-1.06] ; 0.0001 * * *$ & 74.75 & $1.02[1.02-1.03] ;<0.0001 * * *$ & 73.83 \\
\hline$<40$ & $0.71[0.30-1.68] ; 0.4371$ & - & $0.43[0.29-0.62] ;<0.0001 * * *$ & - \\
\hline$[40,50)$ & $0.13[0.02-0.94] ; 0.0433^{*}$ & - & $0.70[0.51-0.98] ; 0.0383^{*}$ & - \\
\hline$[50-60)$ & $0.54[0.25-1.15] ; 0.1120$ & - & $0.97[0.77-1.22] ; 0.7821$ & - \\
\hline$[60-70)$ & $0.98[0.50-1.93] ; 0.9641$ & - & $1.06[0.84-1.34] ; 0.6078$ & - \\
\hline$[70-80)$ & $2.37[1.27-4.42] ; 0.0064 * *$ & - & $1.53[1.20-1.95] ; 0.0005 * * *$ & - \\
\hline$>=80$ & $3.48[1.68-7.22] ; 0.0008 * * *$ & - & $1.89[1.39-2.57] ; 0.0001 * * *$ & - \\
\hline $\begin{array}{l}\text { Charlson standard comorbidity } \\
\text { index }\end{array}$ & $1.21[1.10-1.33] ; 0.0001 * * *$ & 2 & $1.27[1.24-1.31] ;<0.0001 * * *$ & 4 \\
\hline \multicolumn{5}{|l|}{ Past comorbidities } \\
\hline Baseline VT/VF & $2.17[0.30-15.71] ; 0.4444$ & - & $0.76[0.24-2.36] ; 0.6302$ & - \\
\hline Cerebrovascular disease & $4.31[1.83-10.15] ; 0.0008 * * *$ & - & $0.97[0.54-1.71] ; 0.9037$ & - \\
\hline Rheumatic disease & $1.10[0.27-4.53] ; 0.8979$ & - & $0.44[0.20-1.00] ; 0.0486^{*}$ & - \\
\hline
\end{tabular}


Renal diseases

Diabetes mellitus

Hypertension

Malignant dysrhythmia

AMI

COPD

IHD

PVD

Stroke/TIA

Gastrointestinal bleeding

Immune mediated enterocolitis

Anxiety disorder and depression

SCD

Atrial flutter

Accident fall

Malignancy

Metastatic solid tumor

\section{Medications}

ACEI

ARB

Lipid-lowering drugs

Anticoagulants

Insulin

Incretin mimetics

Complete blood counts
$2.14[0.91-5.03] ; 0.0810$ $0.82[0.11-5.95] ; 0.8443$

2.92[1.61-5.28];0.0004***

$2.41[0.58-9.92] ; 0.2246$

$1.07[0.15-7.74] ; 0.9485$

2.61[0.36-18.95];0.3428

1.21[0.51-2.84];0.6669

$2.84[0.69-11.71] ; 0.1488$

$3.91[1.55-9.88] ; 0.0039 * *$

$1.63[0.51-5.23] ; 0.4148$

$0.64[0.16-2.65] ; 0.5425$

$1.08[0.15-7.85] ; 0.9371$

$1.00[0.14-7.26] ; 1.0000 * * *$

$0.00[0.00-$ Inf]; 0.9960

2.82[1.20-6.64];0.0179*

$0.83[0.33-2.10] ; 0.6902$

$0.00[0.00-$ Inf];0.9963

0.95[0.38-2.40];0.9146

$0.40[0.06-2.90] ; 0.3647$

$0.68[0.27-1.70] ; 0.4060$

$1.35[0.19-9.79] ; 0.7664$

$\begin{array}{ll}1.24[0.85-1.82] ; 0.2576 & - \\ 1.43[0.85-2.40] ; 0.1747 & - \\ 1.55[1.23-1.96] ; 0.0002 * * * & - \\ 0.76[0.31-1.83] ; 0.5367 & - \\ 1.35[0.74-2.46] ; 0.3277 & - \\ 1.82[0.81-4.08] ; 0.1462 & - \\ 0.95[0.68-1.32] ; 0.7422 & - \\ 1.04[0.47-2.34] ; 0.9178 & - \\ 0.88[0.47-1.65] ; 0.6858 & - \\ 1.77[1.20-2.62] ; 0.0042 * * & - \\ 0.73[0.45-1.19] ; 0.2046 & - \\ 0.91[0.43-1.91] ; 0.7957 & - \\ 1.49[0.86-2.59] ; 0.1577 & - \\ 0.66[0.16-2.64] ; 0.5556 & - \\ 1.15[0.76-1.76] ; 0.5055 & - \\ 3.27[2.64-4.05] ;<0.0001 * * * & - \\ 6.12[4.75-7.89] ;<0.0001 * * * & - \\ 0.60[0.41-0.90] ; 0.0128 * & - \\ 0.21[0.08-0.56] ; 0.0020 * * & - \\ - & - \\ 0.49[0.33-0.71] ; 0.0002 * * * & - \\ - & - \\ 0.70[0.26-1.87] ; 0.4719 & - \\ & -\end{array}$


Mean corpuscular volume, fL

Eosinophil, x10^9/L

Lymphocyte, $x 10^{\wedge} 9 / \mathrm{L}$

Monocyte, $x 10^{\wedge} 9 / \mathrm{L}$

Neutrophil, $x 10^{\wedge} 9 / \mathrm{L}$

White cell count, $x 10^{\wedge} 9 / \mathrm{L}$

Mean cell haemoglobin, pg

Platelet, $\mathrm{x} 10^{\wedge} 9 / \mathrm{L}$

Red blood count, $\mathrm{x} 10^{\wedge} 12 / \mathrm{L}$

\section{Liver and renal functions}

$\mathrm{K} /$ Potassium, mmol/L

Urate, $\mathrm{mmol} / \mathrm{L}$

Albumin, $\mathrm{g} / \mathrm{L}$

$\mathrm{Na} /$ Sodium, mmol/L

Urea, $\mathrm{mmol} / \mathrm{L}$

Protein, g/L

Creatinine, umol/L

Alkaline phosphatase, U/L

Aspartate transaminase, U/L

Alanine transaminase, $\mathrm{U} / \mathrm{L}$

Bilirubin, umol/L

\section{Lipid and glucose tests}

Triglyceride, $\mathrm{mmol} / \mathrm{L}$

Low-density lipoprotein, mmol/L

High-density lipoprotein, mmol/L
$0.99[0.95-1.03] ; 0.6814$

$1.07[0.36-3.19] ; 0.9060$

$1.13[0.79-1.63] ; 0.5080$

$1.09[0.51-2.31] ; 0.8276$

$0.98[0.88-1.09] ; 0.6727$

$1.08[0.98-1.18] ; 0.1135$

$0.96[0.87-1.07] ; 0.4730$

$1.001[0.998-1.005] ; 0.4143$

$0.70[0.46-1.08] ; 0.1044$

\subsection{7[0.73-1.85];0.5151}

$19.54[0.62-615.16] ; 0.0912$

$0.96[0.92-1.00] ; 0.0656$

$1.02[0.95-1.09] ; 0.6241$

$1.02[1.00-1.05] ; 0.0401 *$

$0.97[0.94-1.01] ; 0.1176$

$1.001[1.000-1.001] ; 0.1034$

$1.00[0.99-1.00] ; 0.3722$

$1.001[1.000-1.002] ; 0.1801$

$1.000[0.999-1.002] ; 0.9668$

$1.00[0.99-1.02] ; 0.6439$

$0.72[0.25-2.11] ; 0.5507$

$1.07[0.61-1.89] ; 0.8039$

$1.17[0.34-3.96] ; 0.8022$
87

0.28

0.67

0.35

3.2

6.7

31.5

181

3.4

4.38

0.46

39.4

134.14

13.32

77.2

97

144

21

40

20.3

1.86

3.25

1.32

$\begin{array}{lll}0.99[0.98-1.01] ; 0.4169 & 97 \\ 0.84[0.52-1.35] ; 0.4723 & 0.02 \\ 0.62[0.50-0.76] ;<0.0001 * * * & 1.1 \\ 1.26[1.06-1.48] ; 0.0072 * * & 0.73 \\ 1.03[1.00-1.07] ; 0.0388^{*} & 6.5 \\ 1.09[1.05-1.12] ;<0.0001 * * * & 8.2 \\ 0.98[0.95-1.02] ; 0.3270 & 29 \\ 1.001[1.000-1.003] ; 0.0422^{*} & 318 \\ 0.71[0.61-0.83] ;<0.0001 * * * & 3.97 \\ & \\ 1.10[0.93-1.32] ; 0.2650 & 4.32 \\ 0.95[0.20-4.56] ; 0.9447 & 0.24 \\ 0.95[0.94-0.97] ;<0.0001 * * * & 37.3 \\ 0.93[0.91-0.95] ;<0.0001 * * * & 137 \\ 1.01[0.99-1.02] ; 0.3426 & 8.26 \\ 0.98[0.96-0.99] ; 0.0003 * * * & 67.6 \\ 1.000[0.999-1.000] ; 0.4670 & 61 \\ 1.00[1.00-1.01] ;<0.0001 * * * & 63 \\ 1.001[1.000-1.001] ; 0.0476 * & 91 \\ 1.000[1.000-1.001] ;<0.0001 * * * 63 \\ 1.00[1.00-1.01] ; 0.1075 & 20.3 \\ 0.73[0.46-1.17] ; 0.1965 & 1.69 \\ 0.97[0.74-1.28] ; 0.8528 & 2.0818 \\ 1.08[0.61-1.93] ; 0.7833 & 1.62 \\ & \end{array}$


Cholesterol, $\mathrm{mmol} / \mathrm{L}$

HbA1c, g/dL

Glucose, mmol/L

Cardiac, clotting, inflammatory,

and acid-base tests

D-dimer, ng/mL

High sensitive troponin-I, ng/L

Lactate dehydrogenase, U/L

APTT, second

Prothrombin time/INR, second

C-reactive protein, $\mathrm{mg} / \mathrm{dL}$

\section{ECG measurements}

Trans QRS initangle

Trans QRS initmag

Trans QRS max angle

Trans QRS max mag

Trans QRS term angle

Trans QRS term mag

Trans QRS cwrot

$P$ wave front axis

$\mathrm{P}$ wave horizon axis

I 40 front axis

I 40 horizon axis

QRS front axis

QRS horizon axis

$1.04[0.65-1.65] ; 0.8796$
$0.87[0.75-1.00] ; 0.0544$
$1.03[0.96-1.11] ; 0.4344$

$1.000[1.000-1.001] ; 0.0639$
$0.99[0.91-1.08] ; 0.8063$
$1.000[0.999-1.002] ; 0.5729$
$1.01[0.98-1.03] ; 0.5725$
$0.99[0.94-1.05] ; 0.8329$
$0.98[0.93-1.04] ; 0.4918$
$1.000[0.996-1.003] ; 0.8833$
$4.73[1.88-11.88] ; 0.0009^{* * *}$
$1.000[0.997-1.003] ; 0.8348$
$0.89[0.46-1.70] ; 0.7180$
$0.99[0.99-1.00] ; 0.0219^{*}$
$1.73[0.45-6.57] ; 0.4224$
$1.01[1.00-1.01] ; 0.0837$
$1.00[0.99-1.01] ; 0.7101$
$1.00[1.00-1.01] ; 0.4592$
$1.00[1.00-1.01] ; 0.3348$
$1.01[1.00-1.01] ; 0.0088^{* *}$
$0.99[0.99-1.00] ; 0.0553$
$1.00[1.00-1.01] ; 0.4944$

\begin{tabular}{l|ll}
5.3 & $0.96[0.77-1.18] ; 0.6876$ & 2.69 \\
10.3 & $0.86[0.82-0.91] ;<0.0001 * * *$ & 14 \\
7.88 & $1.02[0.99-1.05] ; 0.2468$ & 8.29 \\
4712.05 & $1.000[1.000-1.000] ; 0.0406^{*}$ & 779.18 \\
0.0408 & $1.00[0.99-1.02] ; 0.7900$ & 0.0814 \\
355 & $1.001[1.001-1.001] ;<0.0001 * * * 390$ \\
30.7 & $0.99[0.97-1.00] ; 0.0446^{*}$ & 27.7 \\
11 & $1.01[0.99-1.02] ; 0.5410$ & 11.4 \\
0.121 & $1.02[1.00-1.03] ; 0.0631$ & 0.879 \\
17 & & \\
0.467 & $0.999[0.998-1.001] ; 0.3597$ & 13 \\
344 & $0.70[0.43-1.14] ; 0.1530$ & 0.15 \\
1.02 & $1.000[0.999-1.001] ; 0.5058$ & 271 \\
209 & $0.47[0.37-0.59] ;<0.0001 * * *$ & 1.027 \\
0.592 & $0.999[0.997-1.001] ; 0.4206$ & 298 \\
-100 & $0.31[0.15-0.61] ; 0.0007 * * *$ & 0.282 \\
20 & $1.000[0.998-1.003] ; 0.8687$ & -17 \\
44 & $1.001[0.998-1.004] ; 0.5060$ & 15 \\
70 & $0.999[0.997-1.001] ; 0.3760$ & 53 \\
15 & $0.999[0.996-1.002] ; 0.5154$ & 10 \\
14 & $1.000[0.997-1.003] ; 0.9221$ & 16 \\
34 & $1.001[0.999-1.003] ; 0.2077$ & 91 \\
$0.999[0.997-1.001] ; 0.4607$ & -15 \\
& & \\
& & \\
& & \\
& & \\
&
\end{tabular}


T wave 40ms front axis

$\mathrm{T}$ wave $40 \mathrm{~ms}$ horizon axis

ST segment front axis

ST segment horizon axis

$\mathrm{T}$ wave front axis

$\mathrm{T}$ wave horizon axis

Atrialrate

Mean ventricular rate

Mean PT interval

Mean PR segment

Mean QRS duration

Mean QT interval

Mean QTc

QT interval dispersion

$1.000[0.996-1.004] ; 0.8801$
$1.00[1.00-1.01] ; 0.0006 * * *$
$1.001[0.997-1.005] ; 0.6429$
$1.00[1.00-1.01] ; 0.1008$
$1.00[1.00-1.01] ; 0.3781$
$1.00[1.00-1.01] ; 0.4924$
$1.00[1.00-1.01] ; 0.5720$
$1.00[0.99-1.01] ; 0.9165$
$1.00[0.99-1.01] ; 0.6172$
$1.00[0.99-1.01] ; 0.6084$
$1.02[1.01-1.03] ; 0.0004 * * *$
$1.00[1.00-1.01] ; 0.1896$
$1.01[1.00-1.01] ; 0.0374 *$
$1.000[0.995-1.005] ; 0.9536$

$-20$

28

$-16$

145

$-15$

66

87

58

147

89

99

354

431

113

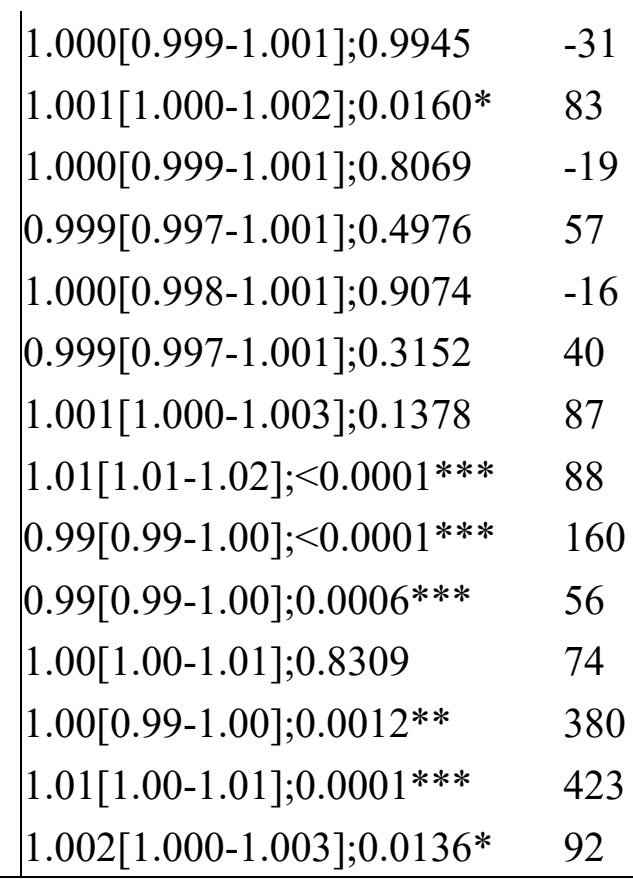

COPD: chronic obstructive pulmonary disease; ACEI: angiotensinogen converting enzyme inhibitor; ARB: angiotensin receptor blocker; APTT: activated partial thromboplastin time. 
915 pericarditis patients with baseline ECG measurements followed up from January $1^{\text {st }}, 2005$ to December $31^{\text {st }}, 2019$

Excluded 41 patients with prior $\mathrm{AF} / \mathrm{HF}$ or with medications for HF.

Study cohort $(\mathrm{N}=874)$ :

396 patients (45.3\%) with all-cause mortality;

52 patients $(5.94 \%)$ with cardiovascular mortality;

57 patients $(6.52 \%)$ developed spontaneous VT/VF;

168 patients $(19.22 \%)$ developed new onset HF;

156 patients $(17.84 \%)$ developed new onset $A F$.

Figure 1. Procedures of data processing. 
medRxiv preprint doi: https://doi.org/10.1101/2021.11.07.21266025; this version posted November 8, 2021. The copyright holder for this preprint (which was not certified by peer review) is the author/funder, who has granted medRxiv a license to display the preprint in perpetuity.

All rights reserved. No reuse allowed without permission.

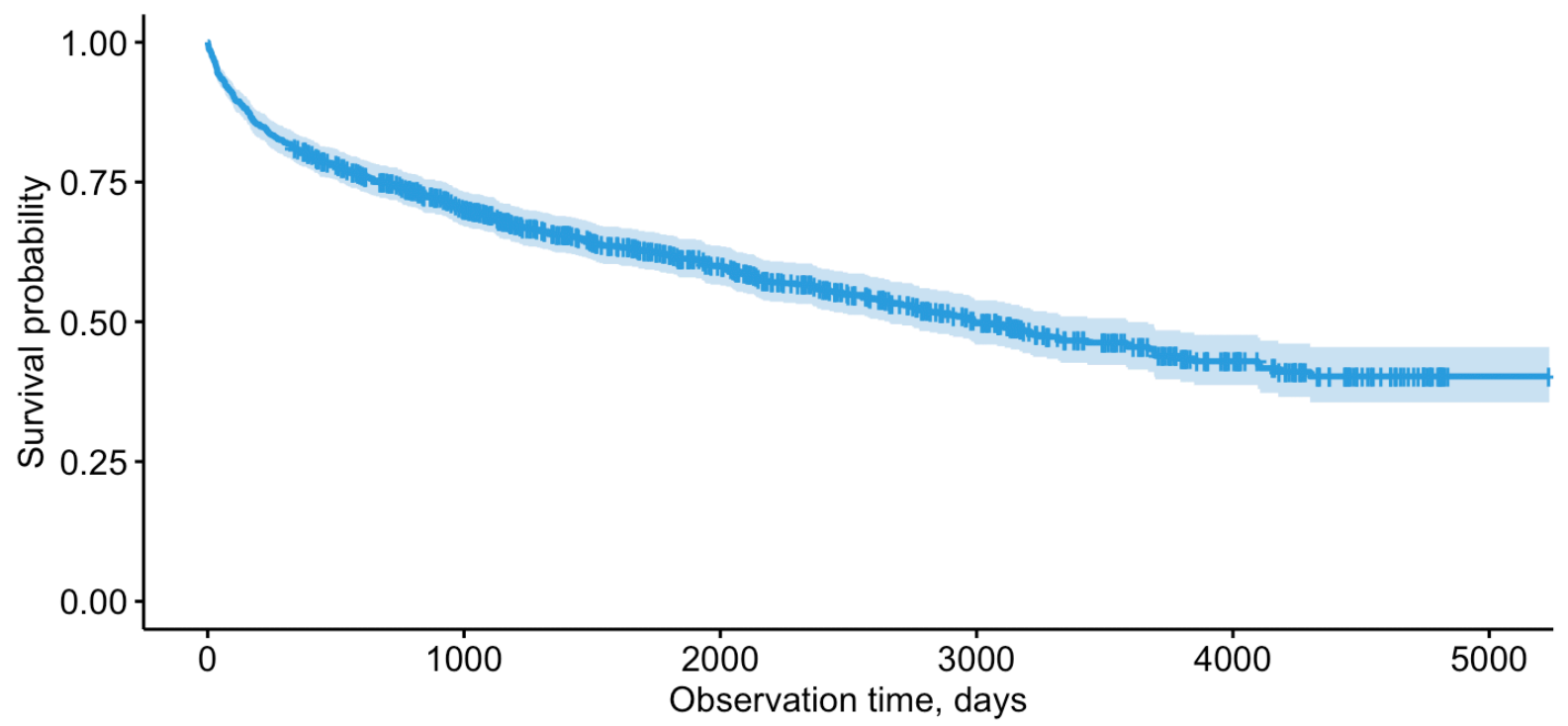

Cardiovascular mortality
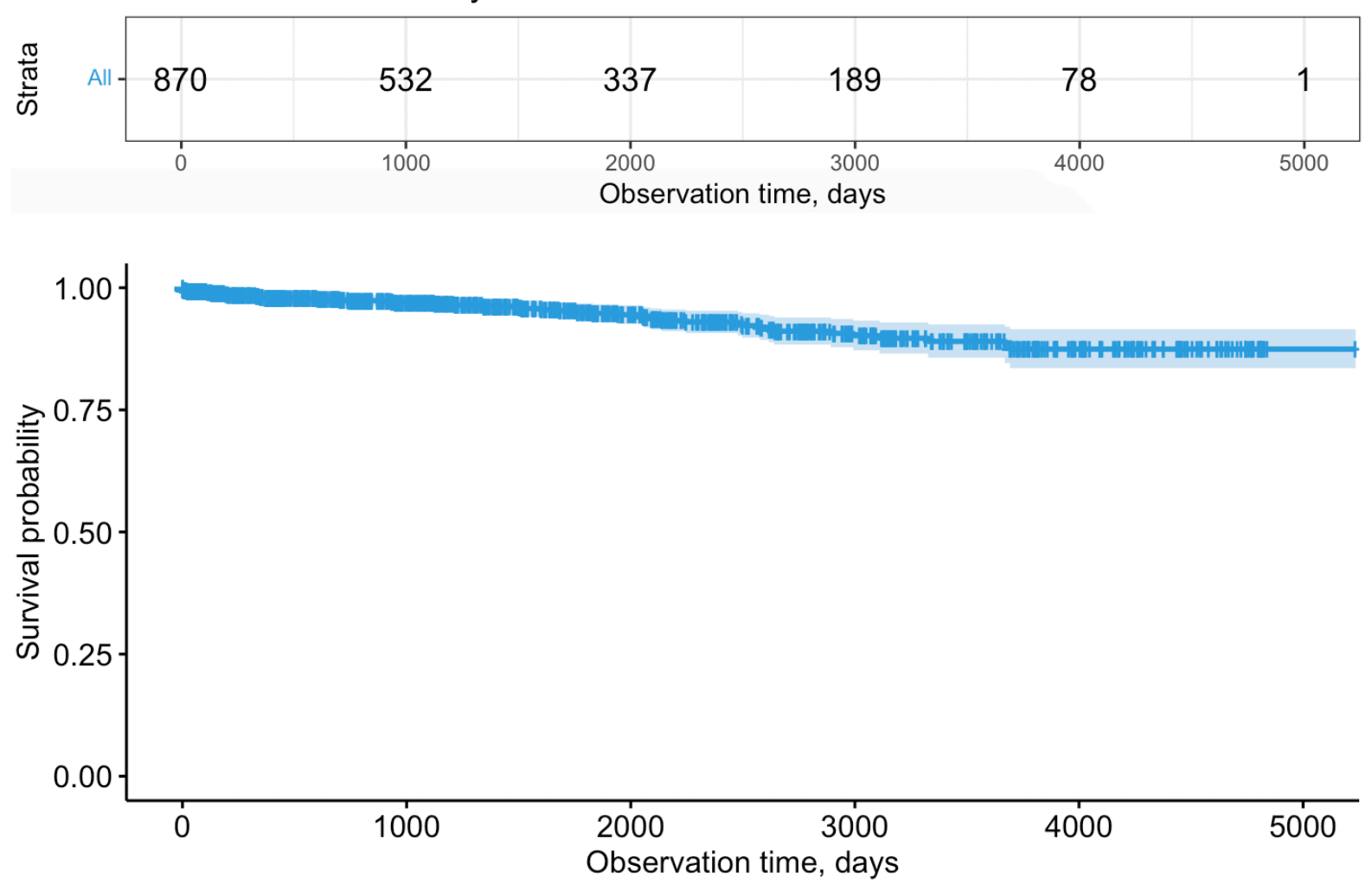

Cardiovascular mortality

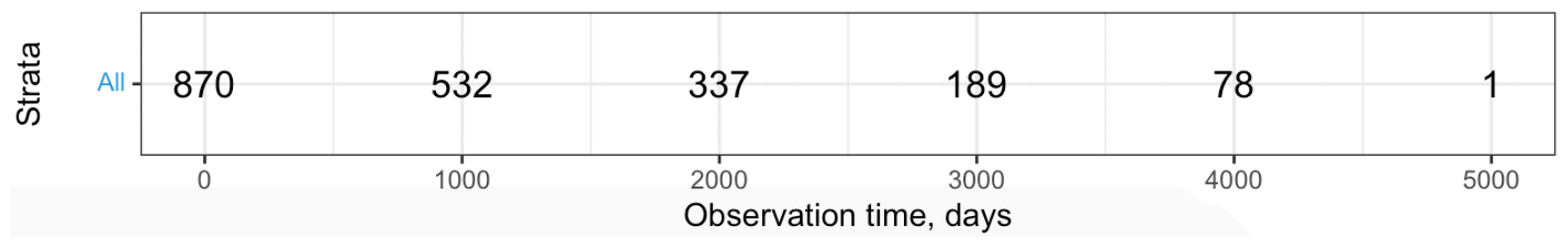


medRxiv preprint doi: https://doi.org/10.1101/2021.11.07.21266025; this version posted November 8, 2021. The copyright holder for this preprint (which was not certified by peer review) is the author/funder, who has granted medRxiv a license to display the preprint in perpetuity.

All rights reserved. No reuse allowed without permission.

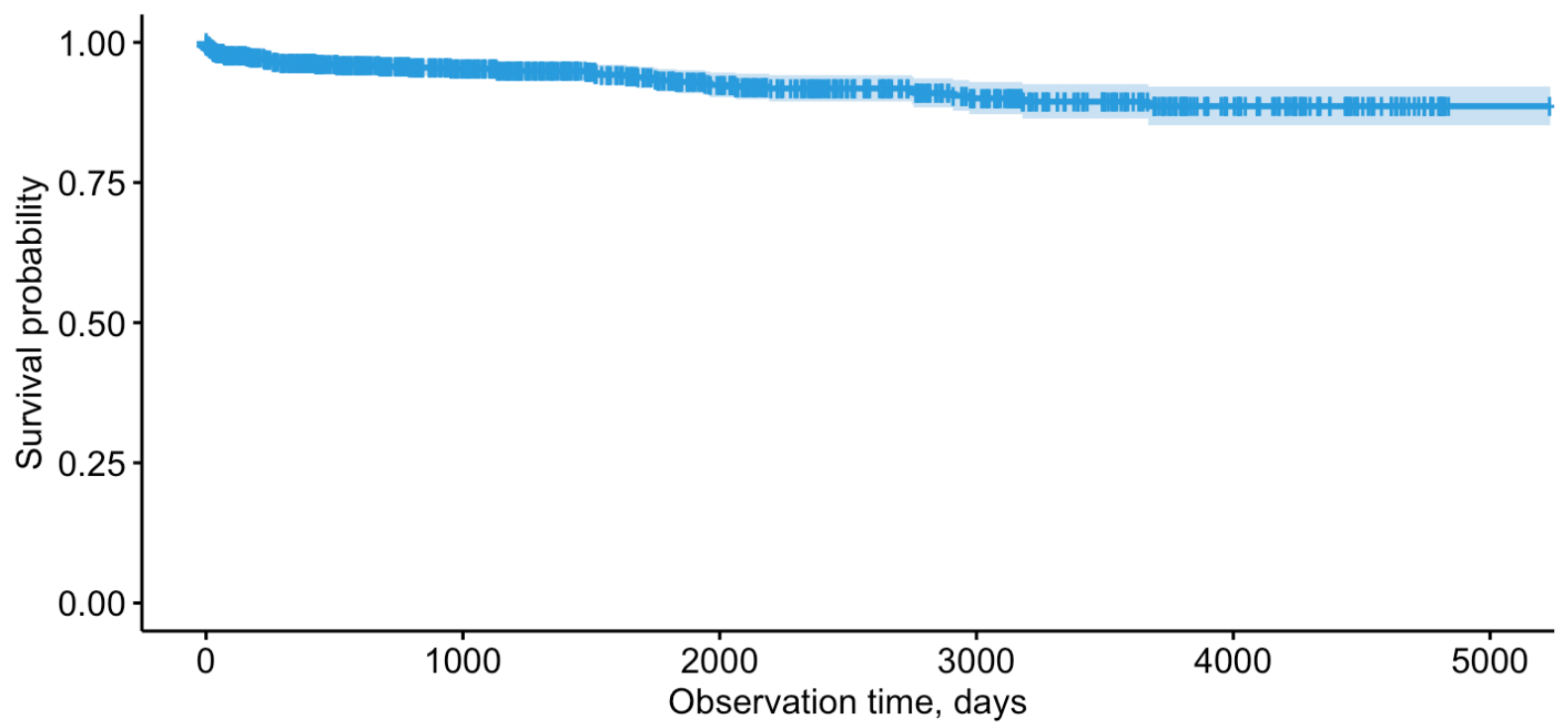

\section{VT/VF}
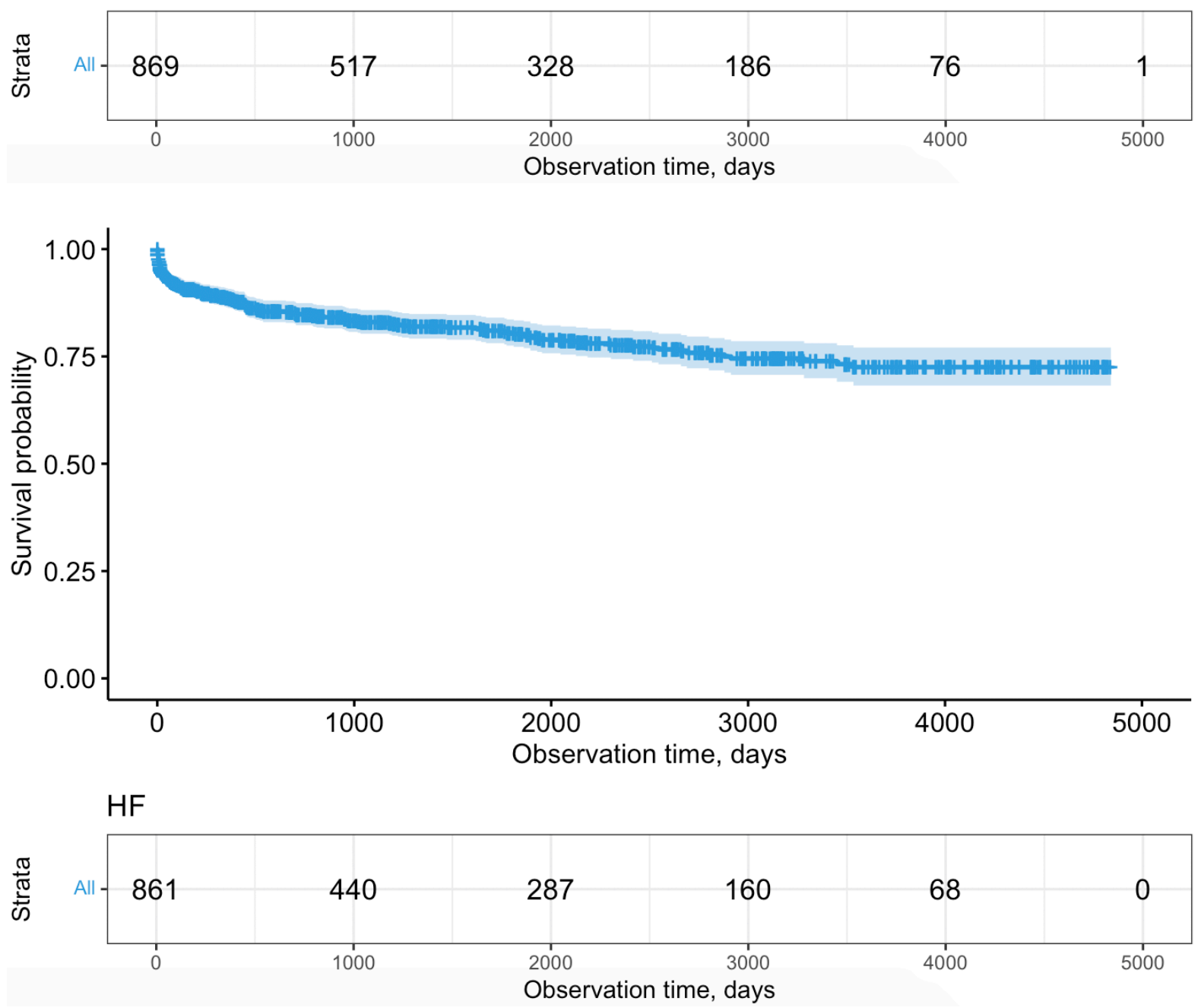
medRxiv preprint doi: https://doi.org/10.1101/2021.11.07.21266025; this version posted November 8, 2021. The copyright holder for this preprint (which was not certified by peer review) is the author/funder, who has granted medRxiv a license to display the preprint in perpetuity.

All rights reserved. No reuse allowed without permission.
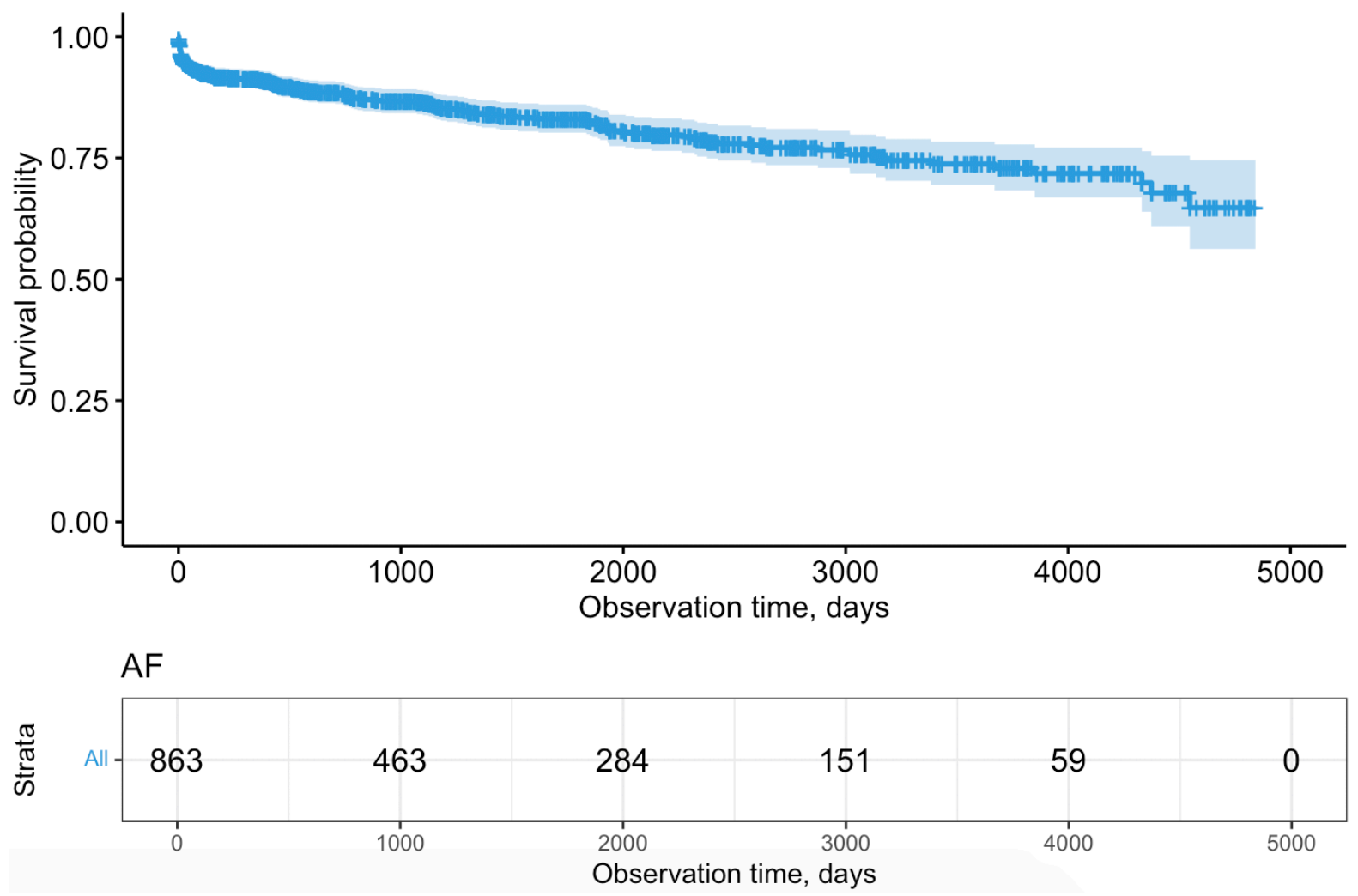

Figure 2. Survival curves of adverse primary and secondary outcomes in pericarditis patients. 


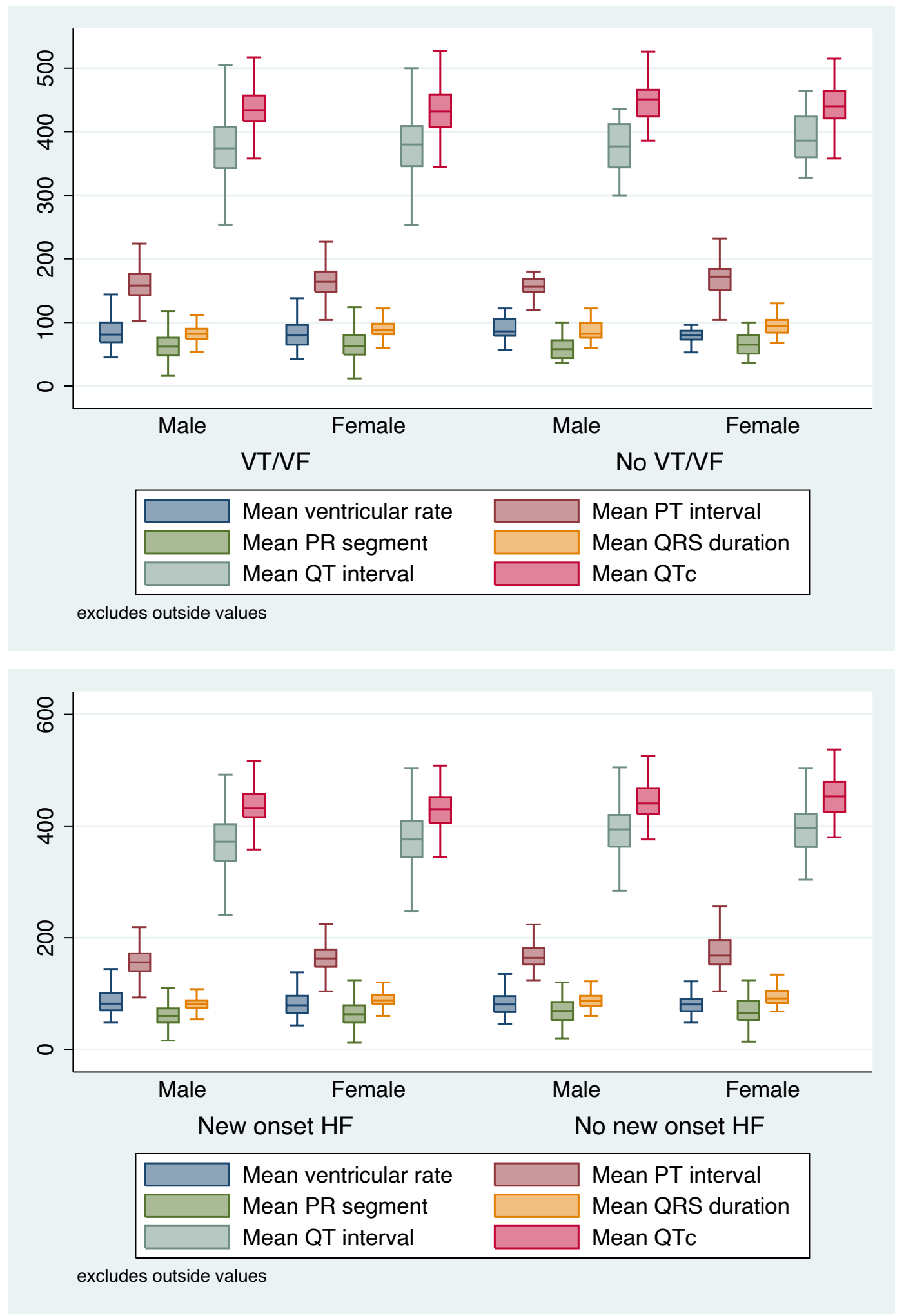




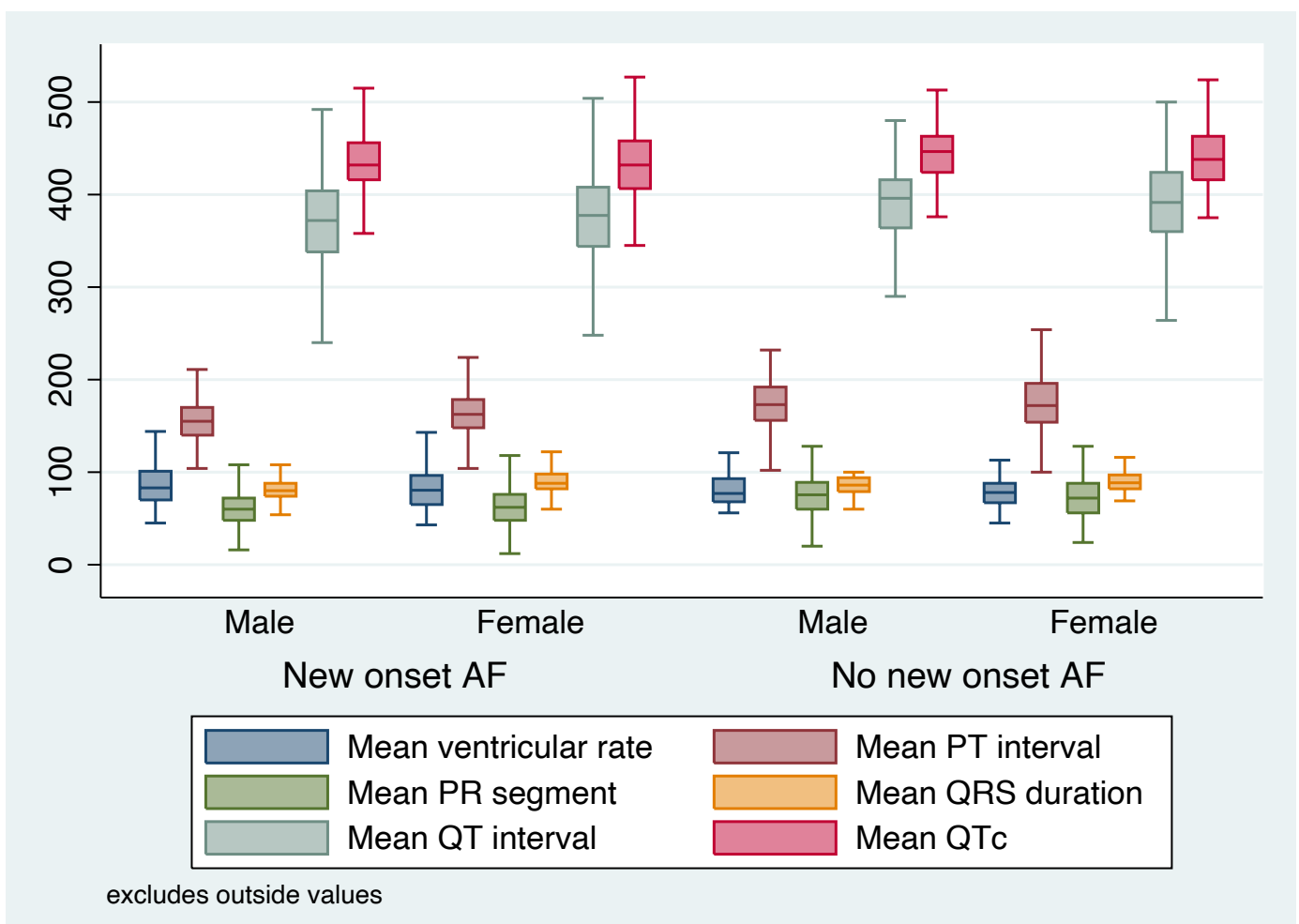

Figure 3. Gender-specific ECG measurements with/without VT/VF, new onset HF and new onset AF. 